\title{
2008/13
}

Modeling international financial returns with a multivariate regime switching copula

Loran Chollete, Andréas Heinen and Alfonso Valdesogo 
CORE

Voie du Roman Pays 34

B-1348 Louvain-la-Neuve, Belgium.

Tel (32 10) 474304

Fax (32 10) 474301

E-mail: corestat-library@uclouvain.be http://www.uclouvain.be/en-44508.html 


\title{
CORE DISCUSSION PAPER \\ $2008 / 13$
}

\section{Modeling international financial returns with a multivariate regime switching copula}

\author{
Loran CHOLLETE $^{1}$, Andréas HEINEN ${ }^{2}$ and Alfonso VALDESOGO ${ }^{3}$
}

\author{
March 2008
}

\begin{abstract}
In order to capture observed asymmetric dependence in international financial returns, we construct a multivariate regime-switching model of copulas. We model dependence with one Gaussian and one canonical vine copula regime. Canonical vines are constructed from bivariate conditional copulas and provide a very flexible way of characterizing dependence in multivariate settings. We apply the model to returns from the G5 and Latin American regions, and document two main findings. First, we discover that models with canonical vines generally dominate alternative dependence structures. Second, the choice of copula is important for risk management, because it modifies the Value at Risk $(\mathrm{VaR})$ of international portfolio returns.
\end{abstract}

Keywords: asymmetric dependence, canonical vine copula, international returns, regime-switching, risk management, Value-at-Risk.

JEL Classification: C32, C35, G10

\footnotetext{
${ }^{1}$ Norwegian School of Economics and Business Administration (NHH), Norway.

${ }^{2}$ Universidad Carlos III de Madrid, Spain. E-mail: aheinen@est-econ.uc3m.es

${ }^{3}$ CORE, Université catholique de Louvain, Belgium. E-mail: alfonso.valdesogo@uclouvain.be
}

We are grateful for comments and suggestions from Jonas Andersson, Luc Bauwens, Victor de la Pena, Rob Engle, René Garcia, Andrew Gelman, Bruno Gerard, Christian Hafner, Malika Hamadi, Philipp Hartmann, Chris Heyde, Bob Hodrick, Jostein Lillestol, Ching-Chih Lu, Thomas Mikosch, Andrew Patton, Jose Scheinkman, Yongzhao Shao, I-Ling Shen, Assaf Zeevi, and participants at NHH, the Norwegian Central Bank, Pace University, Cornell, the Columbia Risk Seminar, Universidad Carlos III de Madrid, ECORE seminar at Université Libre de Bruxelles, the International Conference on Finance in Copenhagen, the Federal Reserve Bank of Boston, and the Federal Reserve Bank of New York, as well as the participants of the Multivariate Volatility Models conference in Faro in October 2007. We acknowledge financial support of the Institut Europlace de Finance. Alfonso Valdesogo acknowledges financial support from the contract "Projet d'Actions de Recherche Concertées" no 07/12-002 of the "Communauté française de Belgique", granted by the "Académie universitaire Louvain". The usual disclaimers apply.

This paper presents research results of the Belgian Program on Interuniversity Poles of Attraction initiated by the Belgian State, Prime Minister's Office, Science Policy Programming. The scientific responsibility is assumed by the authors. 



\section{Introduction}

International financial returns tend to exhibit asymmetric dependence. ${ }^{1}$ This asymmetry means that in times of crisis returns tend to be more dependent than in good times. This phenomenon has important implications for the risk of an international portfolio. In particular, it implies that due to increased dependence in bad times, investors might lose the benefits of diversification when such benefits are most valuable. Hence, international portfolios may be more risky than they seem. The presence of such asymmetric dependence adds a cost to diversifying with foreign stocks, and therefore provides a possible explanation for home bias.

In this paper, we provide further evidence on asymmetric dependence in international financial returns by estimating a regime-switching copula model for the dependence of the stock indices of the G5 and of four Latin American countries. Our contribution is threefold. First, we use regime switching copulas, which allows us to model the dependence in a much more flexible and realistic way than switching models based on the Gaussian distribution, that have been previously proposed, eg. Pelletier (2006). The use of copulas makes it possible to separate the dependence model from the marginal distributions. Copulas also allow us to have tail dependence, which means that, unlike with the Gaussian copula, the dependence does not vanish as we consider increasingly negative returns. Second, we apply this model in a multivariate context, a step towards making this approach feasible for realistic applications. Third, we use a canonical vine copula, a new type of copula that was introduced in finance by Aas, Czado, Frigessi \& Bakken (2007) and which allows for very general types of dependence. Flexibly modeling dependence is very easy with bivariate data, but much more difficult for higher dimensions, given that the choice of copulas is usually thought to be reduced to the Gaussian or the Student t. Both of these copulas are useful only for capturing elliptical dependence. The Gaussian copula suffers from the drawback that it lacks tail dependence, and the multivariate Student $\mathrm{t}$ copula is too restrictive in the sense that, while it can generate different tail dependence for each pair of variables (since the tail dependence is a function of the correlation and the degrees of freedom parameter), it restricts the upper and lower tail dependence for each pair to be the same. While the assumption of tail independence is acceptable for positive returns it is clearly not for negative returns. Canonical vine copulas allow us to to overcome these limitations.

Our paper is related to extant research in at least two areas, asymmetric dependence and regime-switching models, to which we now turn. Regarding asymmetric dependence, Longin \& Solnik (1995) analyze correlations between stock markets over a period of 30 years using the constant conditional correlation (CCC) model of Bollerslev (1990). They find evidence that correlations are not constant and tend to increase over their sample period. Moreover, they are typically higher during more volatile periods and depend on some economic variables such as dividend yields and interest rates. Longin \& Solnik (2001) use extreme value theory and the method of Ledford \& Tawn (1997) to document that extreme correlation, defined as the correlation that exists between returns that are above a certain threshold, are different for positive and negative returns. Ang \& Chen (2002)

\footnotetext{
${ }^{1}$ For evidence on asymmetry, see Longin \& Solnik (1995), Longin \& Solnik (2001), Ang \& Chen (2002), Ang \& Bekaert (2002a), Das \& Uppal (2004) and Patton (2004), amongst others.
} 
develop a test for asymmetric correlation that is based on comparing empirical and modelbased conditional correlations. Amongst the models they compare, regime-switching models are best at replicating this phenomenon. Ang \& Bekaert (2002a) estimate a Gaussian Markov switching model for international returns and identify two regimes, a bear regime with negative returns, high volatilities and correlations and a bull regime with positive mean, low volatilities and correlations. Patton (2004) finds significant asymmetry in the dependence of financial returns both in the marginal distributions and in the dependence structure. He finds that knowledge of asymmetric dependence leads to significant gains for an investor with no short-sales constraints. Our model also relates to other approaches using copulas for financial time series. Patton (2006a) and Patton (2006b) introduce theory for the use of conditional copulas and use time-varying models of bivariate dependence coefficients to model foreign exchange series. Jondeau \& Rockinger (2006) propose to model returns with univariate time-varying skewness Skewed-t GARCH models and then to use a time-varying or a switching Gaussian or Student t copula for the dependence between pairs of countries.

Regarding regime-switching models, our paper follows a long tradition in economics. Regime-switching models were introduced in econometrics by Hamilton (1989) and have since been widely applied in finance. For instance Ang \& Bekaert (2002b), Guidolin \& Timmermann (2006a) and Guidolin \& Timmermann (2006b) use regime-switching models for interest rates. Ang \& Bekaert (2002a) and Guidolin \& Timmermann (2005) use a regimeswitching model for international financial returns. Pelletier (2006) uses regime switching in the context of correlation when the marginals are modeled with GARCH, but he stays in the Gaussian framework. His model lies between the constant conditional correlation (CCC) of Bollerslev (1990) and the dynamic conditional correlation (DCC) model of Engle (2002). Our model can be seen as an extension of the Pelletier (2006) model to the non-Gaussian case. We depart from the Gaussian assumption, as it is well known that returns are not Gaussian, while retaining the intuitively appealing features of a regime switching structure for dependence. Instead of relying on the Gaussian assumption we use canonical vines that are flexible multivariate copulas. We also want to separate asymmetry in the marginals from asymmetry in dependence. This cannot be done in a Gaussian switching model. Instead we rely on copulas and use the flexibility they provide in modeling the marginals separately from the dependence structure. We therefore allow the marginal distributions to be different from the normal by using the Skewed t GARCH model of Hansen (1994).

Very recently, researchers have started to combine copulas and regime switching models in bivariate data. Rodriguez (2007) and Okimoto (2007) estimate regime-switching copulas for pairs of international stock indices. Okimoto (2007) focuses on the US-UK pair, whereas Rodriguez (2007) works with pairs of Latin American and Asian countries. They follow the tradition of Ramchand \& Susmel (1998) to impose a structure where variances, means and correlations switch together. Only Garcia \& Tsafak (2007) estimate a regime-switching model in a 4-variable system of domestic and foreign stocks and bonds by using a clever mixture of bivariate copulas to model the dependence between all possible pairs of variables. To summarize our approach, we estimate regime-switching models with one symmetric Gaussian copula regime and either a Gaussian, a Student $t$ or a canonical vine copula regime. We find that canonical vine models perform best in terms of the likelihood, but also in terms of their ability to replicate the exceedance correlation and quantile dependence 
present in the data. Finally we compute the Value at Risk (VaR) and Expected Shortfall (ES) of an equally weighted portfolio for all models and compare them to the all Gaussian model. We find that the VaR and ES of the canonical vine models are substantially higher than for the Student t or Gaussian copula models, which implies that incorrectly using the latter models can lead to underestimating the risk of a portfolio.

The remainder of the paper is organized in the following manner. In Section 2 we present the model. We briefly discuss copulas, general copula-based dependence measures, as well as tail dependence and canonical vine copulas. Then we present the Markov switching model for dependence, as well as the marginal models. Section 3 describes the two-step estimation procedure for the model, the EM algorithm and the standard errors calculation. Section 4 presents the data and results. In Section 5 we evaluate the performance of the various models. Section 6 concludes.

\section{The Model}

In this section, we first provide a brief account of copula theory, copula-based dependence measures and asymmetric dependence. We then discuss canonical vine copulas, which we use to describe the asymmetric dependence regime. Finally, we introduce the Regimeswitching copula and the marginal models.

\subsection{Copulas}

Traditionally in theoretical finance, the question of the dependence between returns has been addressed using Pearson's correlation. This is due in part to the central role of the normal distribution in statistics and of the Capital Asset Pricing Model (CAPM) in finance. The CAPM assumes multivariate normality of returns and measures dependence with correlation. A limitation of Pearson correlation is that it is implicitly based on the assumption of normality, or more precisely it is only a natural measure of dependence in the elliptical family of distributions. The most prominent members of this family are the multivariate Gaussian and Student t distributions. Another limitation of Pearson correlation is that it only measures linear relations and will therefore miss non-linear relations between variables. Consider, for example two variables $X$ and $Y$, where $X \sim \mathcal{N}(0,1)$ and $Y=X^{2}$. In this setup, $\operatorname{Cov}(X, Y)=\operatorname{Cov}\left(X, X^{2}\right)=\operatorname{Skewness}(X)$. Therefore $X$ and $Y$ are uncorrelated, since their covariance is equal to the skewness of $X$, which is 0 , by normality of $X$. Yet, clearly these variables are perfectly dependent. This simple example shows that correlation is not a good measure of dependence in all cases. ${ }^{2}$

In empirical finance, there is a vast body of literature suggesting that financial returns are not normally distributed. Thus, financial returns might display more intricate types of dependence than can be captured by the correlation coefficient. One way of accounting for these more general types of dependence is through the use of copulas. Copulas are a very flexible tool to model patterns of dependence between variables separately from the marginal distributions, and may be used to model the observed dependence between

\footnotetext{
${ }^{2}$ For further examples, see Embrechts, McNeil \& Straumann (2001) who demonstrate where Pearson correlation fails to capture dependence adequately.
} 
financial returns. ${ }^{3}$ Copula theory goes back to the work of Sklar (1959), who showed that, for continuous variables, a joint distribution can be decomposed into its $n$ marginal distributions and a copula, which fully characterizes dependence between the variables. This theorem provides an easy way to form valid multivariate distributions from known marginals that need not be of the same class. For example, it is possible to use a normal, Student or any other marginal, combine them with a copula and get a suitable joint distribution, which reflects the kind of dependence present in the series. ${ }^{4}$ Specifically, let $H\left(y_{1}, \ldots, y_{n}\right)$ be a continuous $n$-variate cumulative distribution function with univariate margins $F_{i}\left(y_{i}\right)$, $i=1, \ldots, n$, where $F_{i}\left(y_{i}\right)=H\left(\infty, \ldots, y_{i}, \ldots, \infty\right)$. According to Sklar (1959), there exists a function $C$, called a copula, mapping $[0,1]^{n}$ into $[0,1]$, such that:

$$
H\left(y_{1}, \ldots, y_{n}\right)=C\left(F_{1}\left(y_{1}\right), \ldots, F_{n}\left(y_{n}\right)\right) .
$$

The joint density function is given by the product of the marginals and the copula density:

$$
\frac{\partial H\left(y_{1}, \ldots, y_{n}\right)}{\partial y_{1} \ldots \partial y_{n}}=\prod_{i=1}^{K} f_{i}\left(y_{i}\right) \frac{\partial C\left(F_{1}\left(y_{1}\right), \ldots, F_{n}\left(y_{n}\right)\right)}{\partial F_{1}\left(y_{1}\right) \ldots \partial F_{n}\left(y_{n}\right)} .
$$

This allows us to define the copula as a multivariate distribution with Uniform $[0,1]$ margins:

$$
C\left(z_{1}, \ldots, z_{n}\right)=H\left(F_{1}^{-1}\left(z_{1}\right), \ldots, F_{n}^{-1}\left(z_{n}\right)\right),
$$

where $z_{i}=F_{i}\left(y_{i}\right), i=1, \ldots, n$ are the probability integral transformations (PIT) of the marginal models.

Evidently, with the use of copulas we can map the univariate marginal distributions of $n$ random variables, each supported in the $[0,1]$ interval, to their $n$-variate distribution, supported on $[0,1]^{n}$. This method applies, regardless of the type and degree of dependence among the variables.

\subsubsection{Copula based dependence measures}

In order to describe dependence that exists amongst variables that are not in the class of elliptical distributions, there exist several measures, based on ranks of the variables. These measures are invariant with respect to any strictly increasing transformation of the data. Rank correlations are popular distribution-free measures of the association between variables. Unlike the traditional Pearson correlation, they work outside the range of the spherical and elliptical distributions and can detect certain types of non-linear dependence. The two most commonly used coefficients of rank correlation are Kendall's $\tau$ and Spearman's $\rho$. Both rely on the notion of concordance. Intuitively a pair of random variables is concordant whenever large values of one variable are associated with large values of the other variable. More formally, if $\left(y_{i}, x_{i}\right)$ and $\left(y_{j}, x_{j}\right)$ are two observations of random variables $(Y, X)$, we say that the pairs are concordant whenever $\left(y_{i}-y_{j}\right)\left(x_{i}-x_{j}\right)>0$, and discordant whenever $\left(y_{i}-y_{j}\right)\left(x_{i}-x_{j}\right)<0$.

\footnotetext{
${ }^{3}$ For related work on copulas as a modeling tool for returns, see Embrechts, Klüppelberg \& Mikosch (1997), and Dias \& Embrechts (2004).

${ }^{4}$ A more detailed account of copulas can be found in Joe (1997), Nelsen (1999) and in Cherubini, Luciano \& Vecchiato (2004) who provide a more finance-oriented presentation.
} 
Kendall's $\tau$ is defined as the difference between the probability of concordance and the probability of discordance. In general, it can be shown that the Kendall's $\tau$ between variables $X$ and $Y$ can be obtained as $\tau_{X, Y}=\frac{c-d}{c+d}$, where $c$ is the number of concordant pairs and $d$ is the number of discordant pairs. By definition, we then have that the total number of pairs is equal to the number of possible pairs with a sample of $m$ bivariate observations $c+d=\frac{m !}{(m-2) ! 2 !}$. Kendall's $\tau$ can also be expressed as a function of the copula:

$$
\tau=4 \int_{[0,1]^{2}} C(u, v) d C(u, v)-1 .
$$

\subsubsection{Asymmetric dependence, exceedance correlation and tail dependence}

An important feature of financial data is asymmetric dependence. There exist several measures that quantify this feature. In finance, it is of interest to measure both the usual sort of dependence between returns in the center of the distribution, and dependence amongst extreme events. The Normal distribution captures the former, but risk theory deals mostly with the latter, as it is the negative extreme values in the distribution of asset returns that are crucial. There is a fairly large recent literature that studies this sort of extremal dependence. For example, Longin \& Solnik (2001), Ang \& Chen (2002) and Patton (2006a) amongst others use exceedance correlation, which is defined as the correlation between two variables $y_{1}$ and $y_{2}$, conditional on both variables being above or below certain thresholds $\theta_{1}$ and $\theta_{2}$, respectively. Formally, lower exceedance correlation is defined as:

$$
\operatorname{Corr}\left(y_{1}, y_{2} \mid y_{1} \leq \theta_{1}, y_{2} \leq \theta_{2}\right) .
$$

The main findings of these studies is that financial returns tend to exhibit excess correlation in bear markets, but not in bull markets. A Gaussian distribution cannot reproduce this feature. Therefore, while a Gaussian copula with Gaussian margins is unable to generate any exceedance correlation, an asymmetric copula with the same Gaussian marginals can produce this phenomenon. Figure 1 plots the exceedance correlation of a Gaussian and a Rotated Gumbel copula that imply the same level of dependence, as measured by a Kendall's $\tau$ of 0.5 . The data simulated from the Rotated Gumbel copula implies a very different pattern of exceedance correlation than the normal data, which shows that the copulas with lower tail dependence are able to replicate this phenomenon. Exceedance correlation, however, is not without problems. A weakness of this measure is that, like the Pearson correlation, it is not independent of the marginal distributions. Moreover, it is computed only from those observations that are below (above) the threshold, which means that, as we move further out into the tails, the exceedance correlation is measured less and less precisely.

Quantile dependence is a somewhat different measure of the dependence in the tails of the distribution. If $X$ and $Y$ are random variables with distribution functions $F_{X}$ and $F_{Y}$, then there is quantile dependence in the lower tail at threshold $\alpha$, whenever $P[Y<$ $\left.F_{Y}^{-1}(\alpha) \mid X<F_{X}^{-1}(\alpha)\right]$ is different from zero. Finally, tail dependence obtains as the limit of this probability, as we go arbitrarily far out into the tails. The coefficient of lower tail dependence of $X$ and $Y$ is: 


$$
\lim _{\alpha \rightarrow 0^{+}} P\left[Y<F_{Y}^{-1}(\alpha) \mid X<F_{X}^{-1}(\alpha)\right]=\lambda_{L},
$$

provided a limit $\lambda_{L} \in[0,1]$ exists. If $\lambda_{L} \in(0,1], X$ and $Y$ are said to be asymptotically dependent in the lower tail; if $\lambda_{L}=0$ they are asymptotically independent. If the marginal distributions of random variables $X$ and $Y$ are continuous, then the tail dependence of these random variables is a function only of their copula, and hence the amount of tail dependence is invariant under strictly increasing transformations. If a bivariate copula $C$ is such that the limit

$$
\lim _{u \rightarrow 0^{+}} C(u, u) / u=\lambda_{L}
$$

exists, then $C$ has lower tail dependence if $\lambda_{L} \in(0,1]$ and no lower tail dependence if $\lambda_{L}=0$. Similarly, if a bivariate copula $C$ is such that

$$
\lim _{u \rightarrow 1-} \bar{C}(u, u) /(1-u)=\lambda_{U}
$$

exists, then $C$ has upper tail dependence if $\lambda_{U} \in(0,1]$ and no upper tail dependence if $\lambda_{U}=0 . \quad \bar{C}(u, v)=1-u-v+C(1-u, 1-v)$ denotes the survivor function of copula $C$. We list all the copulas we use in this paper along with their tail coefficients in the Appendix. The Gaussian copula does not exhibit any tail dependence except in the case of perfect correlation, where tail dependence equals unity. The Student-t copula exhibits the same amount of upper and lower tail dependence, which is a function of both the degrees of freedom parameter and the correlation coefficient. The Clayton and the Rotated Gumbel have lower, but no upper tail dependence.

\subsubsection{Canonical Vine copula}

We now describe the family of copulas that we use in this paper for the asymmetric regime. Bedford \& Cooke (2002) introduced canonical vine copulas in statistics. These copulas were first used in finance by Aas et al. (2007) and Berg \& Aas (2007), whose presentation we follow here. These general multivariate copulas are obtained by a hierarchical construction. The main idea is that a general multivariate copula can be decomposed into a cascade of bivariate copulas. It is well known that a joint probability density function of $n$ variables $y_{1}, \ldots, y_{n}$ can be decomposed without loss of generality by iteratively conditioning, as follows:

$$
f\left(y_{1}, \ldots, y_{n}\right)=f\left(y_{1}\right) \cdot f\left(y_{2} \mid y_{1}\right) \cdot f\left(y_{3} \mid y_{1}, y_{2}\right) \ldots f\left(y_{n} \mid y_{1}, \ldots, y_{n-1}\right) .
$$

Each one of the factors in this product can be decomposed further using conditional copulas. For instance the first conditional density can be decomposed as:

$$
f\left(y_{2} \mid y_{1}\right)=c_{12}\left(F_{1}\left(y_{1}\right), F_{2}\left(y_{2}\right)\right) f_{2}\left(y_{2}\right) .
$$

Similarly, one possible decomposition of the second conditional density is:

$$
f\left(y_{3} \mid y_{1}, y_{2}\right)=c_{23 \mid 1}\left(F_{2 \mid 1}\left(y_{2} \mid y_{1}\right), F_{3 \mid 1}\left(y_{3} \mid y_{1}\right)\right) f\left(y_{3} \mid y_{1}\right) \text {. }
$$


Further decomposing $f\left(y_{3} \mid y_{1}\right)$ leads to:

$$
f\left(y_{3} \mid y_{1}, y_{2}\right)=c_{23 \mid 1}\left(F_{2 \mid 1}\left(y_{2} \mid y_{1}\right), F_{3 \mid 1}\left(y_{3} \mid y_{1}\right)\right) c_{13}\left(F_{1}\left(y_{1}\right), F_{3}\left(y_{3}\right)\right) f_{3}\left(y_{3}\right) .
$$

Finally, combining the last expressions, one obtains the joint density of the first three variables in the system as a function of marginals and bivariate conditional copulas:

$$
\begin{aligned}
f\left(y_{1}, y_{2}, y_{3}\right)= & c_{23 \mid 1}\left(F_{2 \mid 1}\left(y_{2} \mid y_{1}\right), F_{3 \mid 1}\left(y_{3} \mid y_{1}\right)\right) . \\
& c_{12}\left(F_{1}\left(y_{1}\right), F_{2}\left(y_{2}\right)\right) c_{13}\left(F_{1}\left(y_{1}\right), F_{3}\left(y_{3}\right)\right) f_{1}\left(y_{1}\right) f_{2}\left(y_{2}\right) f_{3}\left(y_{3}\right)
\end{aligned}
$$

Assuming that the marginals are all uniform on the $[0,1]$ interval, the copula can be written as:

$$
c\left(y_{1}, y_{2}, y_{3}\right)=c_{23 \mid 1}\left(F_{2 \mid 1}\left(y_{2} \mid y_{1}\right), F_{3 \mid 1}\left(y_{3} \mid y_{1}\right)\right) c_{12}\left(F_{1}\left(y_{1}\right), F_{2}\left(y_{2}\right)\right) c_{13}\left(F_{1}\left(y_{1}\right), F_{3}\left(y_{3}\right)\right) .
$$

Conditional distribution functions are computed using (Joe (1996)):

$$
F(y \mid v)=\frac{\partial C_{y, v_{j} \mid v_{-j}}\left(F\left(y \mid v_{-j}\right), F\left(v_{j} \mid v_{-j}\right)\right.}{\partial F\left(v_{j} \mid v_{-j}\right)}
$$

In the development above, we have implicitly chosen to condition on $y_{1}$. This choice is arbitrary, and other ways of ordering the data when conditioning are also possible. The choice we have made leads to a canonical vine, in which one variable plays a pivotal role, in our example, $y_{1}$. In the first stage of the copula we model the bivariate copulas of $y_{1}$ with all other variables in the system. Then we condition on $y_{1}$, and consider all bivariate conditional copulas of $y_{2}$ with all other variables in the system etc. For an $n$-dimensional set of variables, this leads to the general $n$-dimensional canonical vine copula:

$$
c\left(y_{1}, \ldots, y_{n}\right)=\prod_{j=1}^{n-1} \prod_{i=1}^{n-j} c_{j, j+i \mid 1, \ldots, j-1}\left(F\left(y_{j} \mid y_{1}, \ldots, y_{j-1}\right), F\left(y_{j+i} \mid y_{1}, \ldots, y_{j-1}\right)\right) .
$$

Figure 2 represents the dependence structure of a canonical vine copula graphically. The advantages of a canonical vine copula are immediately apparent: whereas there are only very few general multivariate copulas, there exists an almost unlimited number of bivariate copulas. When specifying the canonical vine copula, we can therefore choose each one of the building blocks involved from a very long list, which allows a very large number of possible copulas. This reverses the traditional problem of not having enough parametric multivariate copulas to a challenge of having too many to choose from.

It is important to note that some parameters of the canonical vine copula correspond to coefficients of conditional dependence, and are not directly comparable with coefficients, of say, the Gaussian or the Student t copula. Nevertheless, it is possible to express the Gaussian or the Student t copulas in terms of a canonical vine. If all conditional copulas are Gaussian, then the canonical vine coincides with the multivariate Gaussian copula. This is true up to a reparameterization: the correlation matrix of the Gaussian copula contains unconditional correlations, whereas some parameters of the canonical vine copula refer to conditional correlations. One can easily go from one to another using the well-known rules 
of conditional correlation. The corresponding unconditional correlations are obtained by normalizing the unconditional variance covariance matrix obtained via the formula:

$$
\Sigma_{x \mid y}=\Sigma_{x}-\Sigma_{x y} \Sigma_{y}^{-1} \Sigma_{y x}
$$

In the Student t copula, the conditional correlations work in the same way as for the Gaussian, but the degrees of freedom have to be incremented by one, every time one conditions on an additional variable. In order to facilitate comparison across regimes and across models we express our results in terms of the unconditional Kendall's $\tau$. We use the fact that Kendall's $\tau$ is a known function of the copula, as in Equation (4). Furthermore, there exist closed-form solutions for many families of copulas. With this information in hand, we first compute the Kendall's $\tau$ of each bivariate conditional copula implied by the estimated parameter using Equation (4). Then we presume the data came from a Gaussian copula and we compute the copula correlation that implies the same Kendall's $\tau$, via the relation $\sin (\tau \pi / 2)$. Consequently, we can apply the rules of conditional variance-covariance and compute the corresponding unconditional correlations. Finally we report the unconditional Kendall's $\tau$ that corresponds to the unconditional correlation with the relation $2 \arcsin (\rho) / \pi$. Figure 3 illustrates this procedure. Of course this procedure involves some approximation. ${ }^{5}$ Alternatively one could think of transforming the conditional Kendall's $\tau$ to an unconditional one by applying the rules of conditional correlation directly to Kendall's $\tau$. However, as shown in Korn (1984), even with a Gaussian joint distribution, where a pair of variables is independent conditionally on the remaining variables, the conditional Kendall's $\tau$ calculated by applying the same rules as for the Pearson correlation is not necessarily equal to zero. This is due to the nonlinear relationship between Pearson correlation and Kendall's $\tau$. We follow Aas et al. (2007) in using the bivariate Gaussian, Student t, Clayton and Gumbel as building blocks for the canonical vine copula. ${ }^{6}$

\subsection{Regime Switching Copula}

In order to model the dependence in our data we use a regime-switching model. We follow Pelletier (2006) and Garcia \& Tsafak (2007) in allowing for two regimes, characterized by differing levels or shapes of dependence. Our dependence model can be thought of as a multivariate extension of the model in Rodriguez (2007) or as an extension to more realistic dependence of the Pelletier (2006) model. We are closer to Pelletier (2006) in the sense that we model the marginal distributions separately from the dependence structure and therefore do not let them depend on the regime. This is consistent with the modeling approach underlying the DCC model of Engle (2002) and Engle \& Sheppard (2001). Garcia \& Tsafak (2007) is the only other paper we are aware of that uses regime-switching copulas for more than two series and they make the same choice that we do. In the remainder of this section we present the copula-switching model which allows different dependence structures over different subsamples.

\footnotetext{
${ }^{5}$ In order to get an idea of the quality of the approximation, we computed Kendall's $\tau$ on 10000 simulations from the canonical vines in Table 4 . The difference between the two approaches appears in the second decimal and is usually around 0.01 . Note that the simulation method, besides its high computational cost, is also an approximation.

${ }^{6}$ For the Gumbel, we use the rotated version, in order to accommodate negative tail dependence in our data.
} 
Following Hamilton (1989), we assume that the $n$-variate process $Y_{t}$ depends on a latent binary variable that indicates the economy's current regime. In our model the regime only affects the dependence structure. Therefore we switch between two density functions to describe the data. The density of the data conditional on being in regime $j$ is:

$$
f\left(Y_{t} \mid Y_{t-1}, s_{t}=j\right)=c^{(j)}\left(F_{1}\left(y_{1, t}\right), \ldots, F_{n}\left(y_{n, t}\right) ; \theta_{c}^{(j)}\right) \prod_{i=1}^{n} f_{i}\left(y_{i, t} ; \theta_{m, i}\right),
$$

where $Y_{t}=\left(y_{1, t}, \ldots, y_{n, t}\right), s_{t}$ is the latent variable for the regime, $c^{(j)}($.$) is the copula in$ regime $j$, with parameter $\theta_{c}^{(j)}, f_{i}($.$) is the density of the marginal distribution of y_{i}$, with parameter $\theta_{m, i}$, and $F_{i}$ is the corresponding distribution function. Notice that $j$ indexes the copula, but not the marginal densities.

As is standard in the literature we assume that the unobserved latent state variable follows a Markov chain with transition probability

$$
\mathbf{P}=\left(\begin{array}{cc}
p_{11} & 1-p_{11} \\
1-p_{22} & p_{22}
\end{array}\right)
$$

where the $p_{i, j}$ represent the probability of moving from state $i$ at time $t$ to state $j$ at time $t+1$.

\subsection{Marginal Model}

In order to take into account the dynamics of the volatility we model the marginal distributions of each one of our returns using the univariate Skewed-t GARCH model of Hansen (1994), which we fit to the demeaned returns. Specifically, our system is expressed as

$$
\begin{gathered}
y_{i, t}=\sqrt{h_{i, t}} \cdot \varepsilon_{t}, \text { for } i=1, \ldots, n . \\
h_{i, t}=\omega_{i}+\alpha_{i} h_{i, t-1}+\beta_{i} \varepsilon_{i, t-1}^{2} \\
\varepsilon_{i, t} \sim \text { Skewed }-t\left(\nu_{i}, \lambda_{i}\right)
\end{gathered}
$$

where the Skewed-t density is given by

$$
g(z \mid \nu, \lambda)= \begin{cases}b c\left(1+\frac{1}{\nu-2}\left(\frac{b z+a}{1-\lambda}\right)^{2}\right)^{-(\nu+1) / 2} & z<-a / b \\ b c\left(1+\frac{1}{\nu-2}\left(\frac{b z+a}{1+\lambda}\right)^{2}\right)^{-(\nu+1) / 2} & z \geq-a / b\end{cases}
$$

The constants $a, b$ and $c$ are defined as:

$$
a=4 \lambda c\left(\frac{\nu-2}{\nu-1}\right), \quad b^{2}=1+3 \lambda^{2}-a^{2}, \quad c=\frac{\Gamma\left(\frac{\nu+1}{2}\right)}{\sqrt{\pi(\nu-2)} \Gamma\left(\frac{\nu}{2}\right)}
$$

A negative $\lambda$ corresponds to a left-skewed density, which means that there is more probability of observing large negative than large positive returns. This is what we expect, since 
it captures the large negative returns associated to market crashes that are the cause of the skewness. We group all parameters of a given country in a vector $\theta_{m, i}=\left(\omega_{i}, \alpha_{i}, \beta_{i}, \nu_{i}, \lambda_{i}\right)$.

\section{Estimation}

First we explain how we estimate the parameters in a two-step procedure that separates the marginals from the dependence structure. Then we provide a brief account of the EM algorithm that we use for the regime-switching copula model, and finally we show how we compute robust standard errors for all the parameters of the model.

\subsection{Two-step estimation}

When estimating the model, we take advantage of the fact that the marginal densities are not regime-dependent, in order to separate the estimation into two steps. The total log likelihood depends on all the data $\mathbf{Y}=\left(Y_{1}^{\prime}, \ldots, Y_{T}^{\prime}\right)^{\prime}$, and is given by

$$
L(\mathbf{Y} ; \theta, \alpha)=\sum_{t=1}^{T} \log f\left(Y_{t} \mid \mathbf{Y}^{\mathbf{t}-\mathbf{1}} ; \theta, \alpha\right),
$$

where $\mathbf{Y}^{\mathbf{t}-\mathbf{1}}=\left(Y_{1}, \ldots, Y_{t}\right)$ denotes the history of the full process. We can therefore decompose this likelihood into one part, $L_{m}$ that contains the marginal densities and another part, $L_{c}$ that contains the dependence structure:

$$
\begin{gathered}
L(\mathbf{Y} ; \theta, \alpha)=L_{m}\left(\mathbf{Y} ; \theta_{m}\right)+L_{c}\left(\mathbf{Y} ; \theta_{m}, \theta_{c}\right) \\
L_{m}\left(\mathbf{Y} ; \theta_{m}\right)=\sum_{t=1}^{T} \sum_{i=1}^{n} \log f_{i}\left(y_{i, t} \mid y_{i}^{t-1} ; \theta_{m, i}\right) \\
L_{c}\left(\mathbf{Y} ; \theta_{m}, \theta_{c}\right)=\sum_{t=1}^{T} \log c\left(F_{1}\left(y_{1, t} \mid y_{1}^{t-1} ; \theta_{m, 1}\right), \ldots, F_{n}\left(y_{n, t} \mid y_{n}^{t-1}, \theta_{m, n}\right) ; \theta_{c}\right)
\end{gathered}
$$

where $y_{i}^{t-1}=\left(y_{i, 1}, \ldots, y_{i, t}\right)$ denotes the history of the variable $i$. The likelihood of the marginal models, $L_{m}$ is a function of the parameter vector $\theta_{m}=\left(\theta_{m, 1}, \ldots, \theta_{m, n}\right)$, that collects the parameters of each one of the $n$ marginal densities $f_{i}$. The copula likelihood depends directly on the vector $\theta_{c}=\left(\theta_{c}^{(1)}, \ldots, \theta_{c}^{(r)}, \alpha\right)$. This vector collects the copula parameters over all $r$ regimes as well as the parameters of the Markov transition probability matrix and the initial probabilities, $\alpha$. It also depends indirectly on the parameters of the marginal densities, through the distribution function $F_{i}$, because $F_{i}$ transforms observations into uniform $[0,1]$ variables that are the input of the copula. The function $c$ denotes the density of the regime switching copula model.

In our application of the model we have to accommodate a large number of parameters. Consider, for example, a Student t GARCH model and a two regime switching model of the G5 region's stock returns, combined with a Gaussian copula in each regime. This system results in $25 \mathrm{GARCH}$ parameters (a constant, an $\mathrm{ARCH}$, a GARCH parameter in addition to the degrees of freedom and the skewness parameters of the $t$ for each of the 5 series), 
10 pairwise copula correlation parameters for each one of two regimes and 3 parameters for the switching regime (an initial probability and two transition probabilities), for a total of 48 parameters. Moreover, there are strong non-linearities in the copula that increase difficulty of estimation. In this context, it is easy to see that a full one-step maximization of the likelihood is not feasible. Fortunately we can rely on a two-step procedure whose properties have been studied by Newey \& McFadden (1994) and that has previously been applied in a similar context. ${ }^{7}$ In a first step, we assume that conditionally on the past, the different series are uncorrelated. This means that there is no contemporaneous correlation:

$$
\hat{\theta}_{m}=\underset{\theta_{m}}{\operatorname{argmax}} L_{m}\left(\mathbf{Y} ; \theta_{m}\right)
$$

This estimation is straightforward, as it does not depend on the regime switching, and in addition, it can be simplified further by noting that we can actually estimate each GARCH model separately:

$$
\hat{\theta}_{m, i}=\underset{\theta_{m, i}}{\operatorname{argmax}} \sum_{t=1}^{T} \log f_{i}\left(y_{i, t} \mid y_{i}^{t-1} ; \theta_{m, i}\right) .
$$

We then collect the coefficients in a vector: $\hat{\theta}_{m}=\left(\hat{\theta}_{m, i}, \ldots, \hat{\theta}_{m, n}\right)$. In a second step we take the parameter estimates of the marginal models as given in order to estimate the parameters of the switching copula:

$$
\hat{\theta}_{c}=\underset{\theta_{c}}{\operatorname{argmax}} L_{c}\left(\mathbf{Y} ; \hat{\theta}_{m}, \theta_{c}\right) .
$$

\subsection{EM Algorithm}

We now turn to the estimation of the regime switching copula model, that is conditional on having consistently estimated the marginal models. Given the fact that the Markov chain $s_{t}$ is not observable we need to use the filter of Hamilton (1989). ${ }^{8}$ Specifically, the filtered system obeys

$$
\begin{gathered}
\hat{\xi}_{t \mid t}=\frac{\hat{\xi}_{t \mid t-1} \odot \eta_{t}}{\mathbf{1}^{\prime}\left(\hat{\xi}_{t \mid t-1} \odot \eta_{t}\right)}, \\
\hat{\xi}_{t+1 \mid t}=P^{\prime} \hat{\xi}_{t \mid t}, \\
\eta_{t}=\left(\begin{array}{c}
c^{(1)}\left(F_{1}\left(y_{1, t} \mid y_{1}^{t-1}\right), \ldots, F_{n}\left(y_{n, t} \mid y_{n}^{t-1}\right) ; \theta_{c}^{(1)}\right) \\
c^{(2)}\left(F_{1}\left(y_{1, t} \mid y_{1}^{t-1}\right), \ldots, F_{n}\left(y_{n, t} \mid y_{n}^{t-1}\right) ; \theta_{c}^{(2)}\right)
\end{array}\right),
\end{gathered}
$$

\footnotetext{
${ }^{7}$ This method is also used with the multivariate Gaussian distribution in the DCC model by Engle (2002) and Engle \& Sheppard (2001), in the RSDC model of Pelletier (2006), in conditional copula modeling by Patton (2006a), and in regime-switching copula estimation by Garcia \& Tsafak (2007).

${ }^{8}$ This section is based on the presentation in Hamilton (1994), Chapter 22, adapted to our copula switching model and to the case of $r=2$ regimes.
} 
where $\hat{\xi}_{t \mid t}$ is the $(2 \times 1)$ vector containing the probability of being in each regime at time $t$, conditional on the observations up to time $t ; \mathbf{1}$ is a $(2 \times 1)$ vector of $1 s$; and $\odot$ denotes the Hadamard product. The $(2 \times 1)$ vector $\hat{\xi}_{t+1 \mid t}$ gives these probabilities at time $t+1$ conditional on observations up to time $t$. The vector $\eta_{t}$ contains the copula density at time $t$, conditional on being in each one of the two regimes. Equation (5) corresponds to a Bayesian updating of the probability of being in either regime given present time observations $\left(\eta_{t}\right)$. Equation (6) consists in doing one forward iteration of the Markov chain. Iterating over both equations from a given starting value $\hat{\xi}_{1 \mid 0}$ and parameter values $\theta_{c}$ of the copula and $\alpha$ of the Markov chain, one obtains the value of the likelihood:

$$
L_{c}\left(\mathbf{Y} ; \theta_{m}, \theta_{c}\right)=\sum_{t=1}^{T} \log \left(\mathbf{1}^{\prime}\left(\hat{\xi}_{t \mid t-1} \odot \eta_{t}\right)\right) .
$$

\subsection{Standard errors of the estimates}

In this section we show how we compute the standard errors of our estimates. We use a two-step procedure that has been studied in a time series copula context by Patton (2006a), but that also underlies the estimation of the DCC model as explained in Engle \& Sheppard (2001). Both cases are applications of general theorems of Newey \& McFadden (1994), which can be invoked to show that under standard regularity conditions, the following result holds:

$$
\sqrt{T}\left(\hat{\theta}-\theta_{0}\right) \stackrel{A}{\sim} N\left(0, A^{-1} B A^{\prime}-1\right)
$$

where

$$
A=\left[\begin{array}{cc}
\nabla_{\theta_{m} \theta_{m}} L_{m}\left(\mathbf{Y} ; \theta_{m}\right) & 0 \\
\nabla_{\theta_{m} \theta_{c}} L_{c}\left(\mathbf{Y} ; \theta_{m}, \theta_{c}\right) & \nabla_{\theta_{c} \theta_{c}} L_{c}\left(\mathbf{Y} ; \theta_{m}, \theta_{c}\right)
\end{array}\right]=\left[\begin{array}{cc}
A_{11} & 0 \\
A_{12} & A_{22}
\end{array}\right],
$$

and

$$
B=\operatorname{var}\left[\sum_{t=1}^{n}\left(n^{-1 / 2} \nabla_{\theta_{m}}^{\prime} L_{m}\left(Y_{t} ; \theta_{m}\right), n^{-1 / 2} \nabla_{\theta_{c}}^{\prime} L_{c}\left(Y_{t} ; \theta_{m}, \theta_{c}\right)\right)\right]=\left[\begin{array}{cc}
B_{11} & B_{12} \\
B_{12} & B_{22}
\end{array}\right] .
$$

If we apply the partitioned inverse formulas, it is apparent that the variance covariance matrix for each one of the GARCH models for the marginal distributions is the usual Bollerslev \& Wooldridge (1992) robust variance covariance matrix. The variance covariance matrix for the regime-switching copula is an expression that depends on all the parameters. This covariance matrix can be consistently estimated by a plug-in estimator, which is what we use to do inference on the coefficients. Our two-step estimator is obviously less efficient than a single-step estimation, but given the size of the problem, it is the only realistically feasible estimation strategy.

In the estimation we first use the EM algorithm to get in the neighborhood of the optimum and then we do a few iterations of a "brute force" numerical maximization. Note that the M-step in this estimation is no longer available in closed form, since we have to estimate the parameters of a copula for which there is no parametric solution. Instead, even in the 
EM algorithm, we have to perform a numerical maximization for every iteration of the algorithm, which somewhat reduces the attractiveness of the EM-algorithm compared to direct numerical maximization. In the numerical optimization we have to reparameterize all coefficients to lie on the real line. Correlation matrices in the multivariate Gaussian or $\mathrm{t}$ copula are parameterized as in Pelletier (2006) in order to guarantee semi-definite positive matrices with ones on the diagonal. Whenever a parameter of some copula is restricted to lie within the $[a,+\infty]$ interval, we estimate instead the coefficient $a+\exp (x)$; if the parameter lies in $[-\infty, b]$ we use $b-\exp (x)$; and if a coefficient lies in $[a, b]$, we use $\frac{a \exp (x)+b \exp (-x)}{\exp (x)+\exp (-x)}$. We also use this rule for the transition probabilities of the Markov chain, with $a=0$ and $b=1$. Standard errors of coefficients are obtained with the delta method.

\section{Data and Results}

In this section we present the results of the estimation. First we present the results for the marginal models, then we discuss the dependence results for the countries of the G5 and of Latin America.

\subsection{Marginal Models}

We apply the Markov-Switching copula model to the weekly returns of equity indices. Our sample comprises two groups of countries: the G5 (Germany, France, the UK, the US and Japan) and Latin America (Brazil, Mexico, Argentina and Chile). The equity indices are daily MSCI price series from 1995 to 2006, where all prices are in US dollars.

In order to avoid introducing artificial dependence due to the difference in closing times of stock exchanges around the globe, we work with Wednesday to Wednesday returns. This gives us a sample of 596 weekly returns from January 3, 1995 to May 30, 2006. We first present some descriptive statistics in Table 1. All series show very clear signs of non-normality with negative skewness except for Japan and Argentina, which have small positive skewness. Further evidence of non-normality is given by the fact that all series have a kurtosis that is well above 3 . The weekly average returns range from $-0.01 \%$ for Japan to $0.23 \%$ for Mexico. The standard deviations of weekly returns are quite different for both groups of countries. They are around 3\% for the G5 and for Latin America, they range from $3.16 \%$ for Chile to $5.30 \%$ for Brazil. Next we show the correlation matrix of the raw data in Table 8. For the G5, the most highly correlated countries are, unsurprisingly, the European countries: Germany-France with a correlation of 0.86 , followed by UK-France and Germany-UK. The US is also correlated with the European countries. Japan is the least correlated to the other countries, its highest correlation being 0.43 , with France. The overall amount of correlation amongst Latin American countries is much lower than amongst the G5 countries. The highest correlations are Brazil-Mexico (0.64) and Brazil-Chile (0.58), followed by Argentina-Brazil and Argentina-Mexico (both with 0.56).

The results of each of the univariate skewed-t GARCH models are presented in Table 3, columns one to four. We can see that the skew coefficient is negative and significant in all series of the G5 with the exception of Japan. In Latin America, only Brazil has a significantly negative skew. Our rationale for including a skew in the marginal distributions is to ensure that any asymmetry we find in the dependence structure truly reflects dependence 
and cannot be attributed to poor modeling of the marginals. The negative skew we find captures the fact that the tails of some of the marginal distributions are typically longer on the left side. This means that large negative returns, as observed during a stock market collapse, are more likely than very good positive returns of the same magnitude. This corroborates the descriptive statistics of the unconditional distributions of our return series. The degrees of freedom parameters of most series is around 8 , which corresponds to tails of the conditional distribution that are somewhat fatter than those of the normal distribution. As a rule of thumb, one can say that it is very difficult to distinguish a t distribution with more than 10 degrees of freedom from a Gaussian. In the G5, the US has the most Gaussian looking returns of all with a degrees of freedom parameter of almost 17 . France has the fattest tails with about 8 degrees of freedom. Latin American countries have fatter tails with coefficients ranging from 6.32 for Argentina to 10.60 for Chile.

A well specified model for the marginals is crucial, because misspecification can result in biased copula parameter estimates, see Fermanian \& Scaillet (2005). Therefore, we apply a battery of goodness of fit (GOF) tests, including three versions of the Kolmogorov-Smirnov test, the Anderson-Darling and Kuiper tests of uniformity of the PIT of the marginal models. We also perform the Berkowitz test, which is a joint test of uniformity and lack of correlation of the PIT, based on transforming the PIT to the normal and testing an AR(1) model against the uncorrelated standard normal. The p-values of the tests are reported in columns five to ten of Table 3. All models pass all the tests, except for the UK in the Berkowitz test. In the same table, columns 11 to 16, we also present the p-values of the Ljung-Box test of autocorrelation in the squared residuals of the skewed-t innovations of the GARCH models. The table shows that each one of the marginal models is well specified, which is not the case when considering Gaussian or Student $t$ innovations in the GARCH specification.

\subsection{Countries of the G5}

We estimate three models for the G5 data. The results are presented in Table 4, Panel A. The first model (columns two to four) has a Gaussian copula in each regime. The results indicate that we have a high and a low dependence regime. The copula correlation coefficient in the more dependent regime is higher for all pairs of countries, which means that the whole G5 together is more dependent when the economy is in that regime. This regime is characterized by some very large correlations. For instance France and Germany have a correlation coefficient of .92, that translates into a Kendall's $\tau$ of 0.74 , which is very high dependence. More generally the highest correlations are between the European countries. We also estimate a model with a Gaussian and a Student-t regime (columns five to seven). The multivariate Student-t regime corresponds to the lower dependence regime. We estimate the degrees of freedom at 23.95, which is quite large and does not correspond to a qualitatively very different picture from the all Gaussian model. A likelihood ratio would clearly reject the Student-t model, as the likelihood increases by no more than 1.44, with only one additional parameter. The difference between the models is that, unlike the Gaussian, the Student-t copula possesses tail dependence, but it implies equal upper and lower tail dependence, which is clearly at odds with the stylized facts. Finally we show the results of a switching model with a Gaussian and a canonical vine regime (columns eight to 
twelve). The class of possible canonical vines is evidently extremely large. We follow Aas et al. (2007) for the specification of the copula. First, we order the variables by decreasing correlations, choosing the variable with largest correlations as the first one to condition on. This leads us to place Germany at the basis of the construction, followed by France, the UK, the US and finally, Japan. By so doing we intend that most of the dependence structure in the copula will be captured in the lower stages of the canonical vine, leaving only very little dependence to be modeled as we move to copulas that are conditional on more countries. We then start estimating models. As we expect to observe mainly lower tail dependence, we start estimating models with all Rotated Gumbel or Clayton copulas. We notice that the parameters of the second stage of the canonical vine are close to their bounds, suggesting that these copulas are not appropriate. In most cases, we find that the Gaussian or the Student-t copula perform well for the conditional copulas. We use likelihood criteria to decide between the Gaussian and the Student-t copula, along with the estimated parameters for the degrees of freedom. When the degrees of freedom of the Student $\mathrm{t}$ are too high, we use the Gaussian. Our preferred model for the G5 has Rotated Gumbel copulas for all the pairs of variables in the first stage and then Gaussian copulas, except for France-Japan, conditional to Germany which is a Student t copula. Although we can strictly speaking not use the likelihood as a criterion for selecting models that are not nested, we nonetheless note that the canonical vine model increases the likelihood by about 6.5 points compared to the Student t model, with the same number of parameters. Of course we can by no means claim that we have chosen the best possible copula, since more theoretical work is needed about model selection of vine copulas in general. But one way of checking that the chosen model is reasonable is to see whether we can capture the quantile dependence or the exceedance correlation that is present in the data. We also note that in the three models all coefficients are statistically significant, except in the conditional copulas of the canonical vine model. Even though some of the individual conditional copula parameters are not significant, we prefer to include these terms, since the model would otherwise unreasonably imply dependence only amongst the variables in the first stage of the canonical vine. In order to check this, we estimate the canonical vine model, where we restrict all the conditional copulas to be independent, and we obtain a loglikelihood of 887.57, which implies a likelihood ratio test statistic of 31.3 for 7 degrees of freedom, which is indicative of a strong rejection of the conditionally independent model. All models for the G5 are characterized by very high persistence in both regimes. When we examine the plot of the smoothed probabilities of being in the high dependence regime, in the first row of Figure 9, we can see that the economy is mostly in the low dependence regime until 1997, whereas the high dependence regime is the dominant one from 1997 onwards. One factor explaining this might be the increased integration between financial markets in Europe, linked to the introduction of the Euro. More generally it seems that since the second part of the nineties, the returns from the G5 have all become much more highly dependent. The smoothed probabilities differ very little from one model to another and the dependence within each regime, as measured by the unconditional Kendall's $\tau$ seems to change very little from one model to another. 


\subsection{Latin American Countries}

We also estimate three models for the group of Latin American countries. The results are presented in Table 4, Panel B. We estimate the same models as for the G5. By contrast, in Latin America the high dependence regime coincides with the asymmetric one. In the all Gaussian copula regime all correlation coefficients are higher in the first regime than in the second one. In the high dependence regime the correlations range from 0.79 for Brazil-Mexico to 0.59 for Argentina-Chile, while for the low dependence regime they range from 0.30 to 0.43. We then estimate a Student t Gaussian copula model. The Student t copula regime has a fairly low number of degrees of freedom. Unlike with G5, a likelihood ratio test would strongly reject the all Gaussian copula model, as we increase the likelihood by 5.74 points by adding just one parameter. Finally we show the results of a switching model with a canonical vine and a Gaussian regime (columns eight to twelve). As in the Student t copula model, the canonical vine copula is in the high dependence regime. In order to select the structure of the canonical vine copula, we have followed the same rules used for the G5. We start estimating models with all Rotated Gumbel or Clayton copulas, then we made modifications in the structure by using different bivariate copulas, such as the Student t, Normal and Gumbel. The final canonical vine structure is in many ways similar to the G5, since the first stage is characterized by otated Gumbel copula for all pairs, and then we have only Gaussian copulas for all conditional copulas. Notice that the canonical vine model increases the likelihood by almost 18 points compared to the Student t model with one parameter less. The transition probability matrix shows fairly high persistence in both regimes for the Student t and canonical vine copula models. In the second row of Figure 9, we plot the smoothed probabilities implied by the three models. Here, one can observe a striking difference between the all-Gaussian model and the other two models. This is reflected also in the transition probabilities of the all Gaussian model that implies much less persistence than the other models. Another way to see this is by comparing the unconditional Kendall's $\tau$ parameters for the three models. While the Student t-Gaussian and Canonical vine-Gaussian models identify regimes with similar dependence, the all Gaussian copula model has more extreme differences between the regimes. The regime switching models with the Student $t$ and the one with the canonical vine copulas seem to identify a first regime, which is the predominant one. This regime features high dependence relative to Argentina, especially in the case of Brazil and Mexico. These two countries have a Kendall's $\tau$ of $0.46,0.45$ in the Student-t model; and 0.43, 0.44 in the canonical vine model, respectively. The second regime is characterized by high dependence relative to Brazil, especially for Mexico and Chile. Now these two countries have Kendall's $\tau$ of 0.44 and 0.49 in model 2 (Student t); and 0.48 and 0.49 in model 3 (canonical vine), respectively. It seems that the all- Gaussian copula model is compensating for the lack of tail dependence in each regime by exaggerating the difference between regimes and switching very often between them depending on the observations. For the Student $t$ and the canonical vine copulas the smoothed probabilities and the dependence within each regime, as measured by the unconditional Kendall's $\tau$ seem to change very little from one model to another. 


\section{Evaluation of the models}

In order to evaluate the models we first analyze their behavior in terms of exceedance correlation and quantile dependence, and then we see what their implications are for value at risk and expected shortfall.

\subsection{Exceedance correlation and Quantile dependence}

One way to evaluate the quality of the model is to provide evidence of the exceedance correlation and quantile dependence implied by the model and compare it with those estimated from the data. Instead of focusing only on tail dependence, we investigate the behavior of the quantile dependence. Examining the behavior of quantile dependence for different thresholds is more informative than concentrating on its asymptotic behavior. We simulate a long series of $N_{b}=298000$ observations from each switching copula model, which corresponds to 500 times our sample size of 596. This yields observations that are uniform. In order to compute the correlation we use the inverse normal cumulative distribution function (cdf) to get values for each return in the real line. With this simulated data we compute exceedance correlation for the following thresholds: from 0.1 to 0.9 by increments of 0.025 . Figures 5 and 6 plot the pairwise empirical exceedance correlations based on the inverse normal cdf of the PIT of the marginals (data) by dots. The reason for these transformations is to remove all asymmetry implied by the marginals. In the same figures we also plot the exceedance correlation of the estimated models of the G5 and of Latin America respectively. The dashed lines represent the all Gaussian copula model, the dot-dashed line is for the Student t copula model, while the continuous line represents the canonical vine model. The plots reveal the presence of asymmetry in the exceedance correlation of the data. This is an indication that it is the underlying dependence structure that is asymmetric, since by construction the marginals are symmetric (we use the normal). Gaussian and Student $\mathrm{t}$ copula models do not fit the asymmetric pattern that we observe of the data. This is due to the fact that both models are based on symmetric copulas. However, the canonical vine model, which has some asymmetric bivariate copulas, does much better at replicating the asymmetry of exceedance correlation implied by the data. For example, for GermanyFrance and Brazil-Argentina the data asymmetry is not captured by the two first models, while the canonical vine model more closely resembles the data. Generally speaking the G5 displays less asymmetry in the exceedance correlation than Latin America, Although this asymmetry is not negligible, as the analysis of the VaR in the next section confirms.

We now proceed to assess whether the estimated models can reproduce the same patterns of quantile dependence as is in the data. Figures 7 and 8 show the pairwise quantile dependence implied by the all Gaussian and the canonical vine copula model, respectively, for Latin America. In both figures the continuous line represents the quantile dependence of the PIT of the marginals of the GARCH models (the data), while the dashed line is the one calculated from simulations of the model. We also plot the $5 \%$ and $95 \%$ confidence intervals represented by lines connecting dots. These confidence intervals are obtained from 500 bootstrap replications of the data. We use the bootstrap method proposed by Caillault \& Guégan (2005) for the selection of the best threshold to estimate tail dependence. We are not using it to select an optimal threshold but simply to have an idea of the variability of 
the estimated quantile dependence. We also plot the average over the bootstrap sample by a dotted line, providing a smoother estimate of the quantile dependence than the in-sample estimate. The plots below the diagonal of each figure represent the lower quantile dependence, while the ones above the diagonal are the upper quantile dependence. One notices the asymmetry between upper and lower quantile dependence of the data (PIT). The lower quantile dependence is in general higher than the upper quantile dependence, which is a stylized fact of international returns. When we consider the models under study, and we compare to the PIT, we find that the all Gaussian copula models tend to underestimate lower quantile dependence and overestimate upper quantile dependence. This is due to the fact that the Normal copula is symmetric while the quantile dependence in the data is not. For example, in the case of Brazil-Mexico, the lower tail dependence implied by the all Gaussian copula model is always below the one implied by the data and sometimes even below the $5 \%$ percentile, while the upper tail dependence is always above the one implied by the data. The canonical vine model tends to fit observed behavior better. For the exceedance correlation, this is due to the fact that some of the bivariate copula components of the canonical vine model are not symmetric. The Rotated Gumbel is asymmetric, implying more dependence in the lower quantile than in the higher quantile. The canonical vine model sometimes overestimates the upper quantile dependence, but to a much lesser degree than the all Gaussian copula model. We find qualitatively similar results for the G5, even though the results are more pronounced for Latin America. There is an asymmetry in the quantile dependence of the data that the canonical vine model is better able to capture than the other models.

\subsection{Value at Risk}

In order to compare the implications of an all Gaussian copula model with a Student $t$ copula and canonical vine model, we compare simulated values of Value at Risk (VaR) and of Expected Shortfall (ES). VaR is one of the most commonly used risk measures for a portfolio. For a given threshold $\alpha, \operatorname{VaR}(\alpha)$ is the $\alpha$ percentile point of the portfolio loss function, and the expected shortfall $E S(\alpha)$ is the expected loss conditional on observing a return below the VaR. Formally, The VaR of a portfolio at the confidence level $\alpha$ is

$$
\operatorname{VaR}(\alpha)=\inf \{l: \operatorname{Prob}(L>l) \leq 1-\alpha\}
$$

and Expected Shortfall (ES) is

$$
E S(\alpha)=E[L \mid L \geq \operatorname{VaR}(\alpha)]
$$

where $L$ is the loss of the portfolio. We use the same simulated series for each model that we have used in the previous section to calculate exceedance correlation. As for the exceedance correlation, we use the inverse normal cdf to obtain values for each return in the real line. ${ }^{9}$ We then form an equally weighted portfolio of all countries in each of our two groups. We use an equally weighted portfolio both for simplicity and because this means that we put equal weight in the bivariate tails for each pair of countries. We thus assign

\footnotetext{
${ }^{9}$ This approach means that the VaR that we calculate is not directly comparable to the one obtained from the real data, but it still allows comparisons between models.
} 
the same weight to the tails of pairs of countries that we model directly in the vine (like dependence with Brazil for Latin American countries), for which one might expect that the model would do better. We do the same for other country pairs. We compute VaR for thresholds between $90 \%$ and $99 \%$ and plot the VaR of the Student-t copula and canonical vine models relative to the all Gaussian switching model. The results, displayed for Latin America in Figure 10, show that use of the all-Gaussian model underestimates the VaR by up $6 \%$ in the empirically relevant case of a $99 \%$ VaR. The Student-t copula model performs close to the all-Gaussian one. Therefore, failing to model the tail dependence in a flexible way can lead to seriously underestimating the VaR for a portfolio. We repeat this analysis with ES, and a similar pattern emerges. Again at a level of 99\%, the ES for the canonical vine model is higher by $7 \%$ with respect to the all Gaussian copula model. We find a similar qualitative picture for the G5, although the results are not quite as dramatic. For the G5, In Figure 9, VaR at $99 \%$ is underestimated by $3 \%$, while ES is around $4.5 \%$ higher with the canonical vine copula model.

\section{Conclusion}

We provide further evidence on asymmetric dependence in international financial returns by estimating a regime-switching copula model for the dependence of the stock indices in the G5 and four Latin American countries. We use regime switching copulas, which allows us to model dependence in a much more flexible and realistic way than previously-suggested switching models based on the Gaussian copula. Moreover, we apply this approach in a multivariate context, thereby taking a step towards making this framework feasible for realistic portfolio applications. In order to model dependence we use a canonical vine copula, which was recently introduced in finance by Aas et al. (2007) and which accommodates general types of dependence. It is based on decomposing a multivariate copula into a product of bivariate iteratively conditional copulas, each of which can be chosen from a long list, producing a large, flexible class of models. Our approach has both econometric and financial aspects, which we summarize.

Regarding econometric implementation, our class of empirical models includes one symmetric Gaussian copula regime combined with either a Gaussian, a Student-t or a canonical copula regime. We find that the canonical vine model dominates, on the basis of the likelihood. The canonical vine models we estimate contain asymmetric copulas in the first level and symmetric copulas (Gaussian or Student t) for the conditional level. We evaluate the models in terms of their ability to replicate the pairwise exceedance correlation and quantile dependence of the data. The canonical vine models are better able to replicate the exceedance correlation in the data. They also dominate in terms of replicating the upper and lower pairwise quantile dependence of the data.

Regarding financial implications, we compute the Value-at- Risk (VaR) and Expected Shortfall (ES) of an equally weighted portfolio for all models and compare them to the all-Gaussian model. We discover that the VaR and ES of the canonical vine models are substantially higher than for the Student-t or Gaussian copula models. This discovery implies that use of our class of models can lead to better estimation of a portfolio's risk. Therefore, in addition to a superior econometric fit, our regime-switching copula approach may yield enhanced performance for financial risk management with large portfolios. 


\section{References}

Aas, K., Czado, C., Frigessi, A. \& Bakken, H. (2007), 'Pair-copula constructions of multiple dependence', Insurance: Mathematics and Economics, forthcoming .

Ang, A. \& Bekaert, G. (2002a), 'International asset allocation with regime shifts', Review of Financial Studies 15(4), 1137-87.

Ang, A. \& Bekaert, G. (2002b), 'Regime switches in interest rates', Journal of Business and Economic Statistics 20, 163-182.

Ang, A. \& Chen, J. (2002), 'Asymmetric correlations of equity portfolios', Journal of Financial Economics 63(3), 443-94.

Bedford, T. \& Cooke, R. M. (2002), 'Vines - a new graphical model for dependent random variables', Annals of Statistics 30(4), 1031-1068.

Berg, D. \& Aas, K. (2007), Models for construction of multivariate dependence, Technical report, Norwegian Computing Center.

Berkowitz, J. (2001), 'Testing density forecasts with applications to risk management', Journal of Business and Economic Statistics 19, 465-474.

Bollerslev, T. (1990), 'Modelling the coherence in short-run nominal exchange rates: A multivariate generalized arch model', Review of Economics and Statistics 72(3), 498505.

Bollerslev, T. \& Wooldridge, J. M. (1992), 'Quasi-maximum likelihood estimation and inference in dynamic models with time-varying covariances', Econometric Reviews 11(2), 143-72.

Caillault, C. \& Guégan, D. (2005), 'Empirical estimation of tail dependence using copulas. application to asian markets', Quantitative Finance 5, 489 - 501.

Cherubini, U., Luciano, E. \& Vecchiato, W. (2004), Copula Methods in Finance, Wiley, Wes Sussex, England.

Das, S. \& Uppal, R. (2004), 'Systemic risk and international portfolio choice', Journal of Finance 59(6), 2809-2834.

Dias, A. \& Embrechts, P. (2004), Dynamic copula models for multivariate high-frequency data in finance, Technical report.

Embrechts, P., Klüppelberg, C. \& Mikosch, T. (1997), Modelling Extremal Events for Insurance and Finance, Springer, New York.

Embrechts, P., McNeil, A. \& Straumann, D. (2001), Correlation and dependence in risk management: Properties and pitfalls, Technical report, Departement Mathematik, ETHZ.

Engle, R. (2002), 'Dynamic conditional correlation: A simple class of multivariate generalized autoregressive conditional heteroskedasticity models', Journal of Business and Economic Statistics 20(3), 339-50.

Engle, R. F. \& Sheppard, K. (2001), Theoretical and empirical properties of dynamic conditional correlation multivariate garch, Technical report, National Bureau of Economic Research Working Paper: 8554. 
Fermanian, J.-D. \& Scaillet, O. (2005), 'Some statistical pitfalls in copula modeling for financial applications', Capital Formation, Governance and Banking pp. 57-72.

Garcia, R. \& Tsafak (2007), Dependence structure and extreme comovements in international equity and bond markets with portfolio diversification effects, Technical report, CIRANO.

Guidolin, M. \& Timmermann, A. (2005), International asset allocation under regime switching, skew and kurtosis preferences, Technical report, Federal Reserve Bank of St. Louis.

Guidolin, M. \& Timmermann, A. (2006a), 'An econometric model of nonlinear dynamics in the joint distribution of stock and bond returns', Journal of Applied Econometrics 21, 1-22.

Guidolin, M. \& Timmermann, A. (2006b), 'Term structure of risk under alternative econometric specifications', Journal of Econometrics 131, 285-308.

Hamilton, J. D. (1989), 'A new approach to the economic analysis of nonstationary time series and the business cycle', Econometrica 57, 357-384.

Hamilton, J. D. (1994), Time Series Analysis, Princeton Press.

Hansen, B. (1994), 'Autoregressive conditional density estimation', International Economic Review 35, 705-730.

Joe, H. (1996), Families, of m-variate distributions with given margins and $\mathrm{m}(\mathrm{m}-1) / 2$ bivariate dependence parameters, in L. Rüschendorf, B. Schweitzer \& M. Taylor, eds, 'Distributions with Fixed Marginals and Related Topics'.

Joe, H. (1997), Multivariate models and dependence concepts, Chapman and Hall/CRC, London; New York.

Jondeau, E. \& Rockinger, M. (2006), 'The copula-garch model of conditional dependencies: An international stock market application', Journal of International Money and Finance 25, 827-853.

Korn, E. L. (1984), 'The ranges of limiting values of some partial correlations under conditional independence', The American Statistician 38(1), 61-62.

Ledford, A. W. \& Tawn, J. A. (1997), 'Statistics for near independence in multivariate extreme values', Biometrika 55, 169-187.

Longin, F. \& Solnik, B. (1995), 'Is the correlation in international equity returns constant: 1960-1990?', Journal of International Money and Finance 14(1), 3-26.

Longin, F. \& Solnik, B. (2001), 'Extreme correlation of international equity markets', Journal of Finance 56(2), 649-76.

Nelsen, R. (1999), An Introduction to Copulas, Lecture Notes in Statistics, Springer-Verlag New York, Inc., New York.

Newey, W. \& McFadden, D. (1994), Large sample estimation and hypothesis testing, in 'Handbook of Econometrics', Vol. 4, North-Holland, Amsterdam, p. 21112245.

Okimoto, T. (2007), 'New evidence of asymmetric dependence structures in international equity markets', Journal of Financial and Quantitative Analysis, forthcoming . 
Patton, A. (2004), 'On the out-of-sample importance of skewness and asymmetric dependence for asset allocation', Journal of Financial Econometrics 2(1), 130-168.

Patton, A. (2006a), 'Estimation of multivariate models for time series of possibly different lengths', Journal of Applied Econometrics 21(2), 147-173.

Patton, A. (2006b), 'Modelling asymmetric exchange rate dependence', International Economic Review 47(2), 527-556.

Pelletier, D. (2006), 'Regime-switching for dynamic correlation', Journal of Econometrics 131, 445473.

Ramchand, L. \& Susmel, R. (1998), 'Volatility and cross correlation across major stock markets', Journal of Empirical Finance 17, 581-610.

Rodriguez, J. (2007), 'Measuring financial contagion: A copula approach', Journal of Empirical Finance 14, 401-423.

Sklar, A. (1959), 'Fonctions de répartition à n dimensions et leurs marges', Pub. Inst. Statist. Univ. Paris 8, 229-231. 


\section{Appendix}

\subsection{Copulas}

\subsubsection{Gaussian copula}

The distribution function of the Gaussian copula is:

$$
C_{N}\left(u_{1}, \ldots, u_{n} ; \Sigma\right)=\Phi_{\Sigma}\left(\Phi^{-1}\left(u_{1}\right), \ldots, \Phi^{-1}\left(u_{n}\right)\right),
$$

where $\Phi^{-1}$ denotes the inverse cumulative density of the standard normal and $\Phi_{\Sigma}\left(x_{1}, \ldots, x_{n} ; \Sigma\right)$ denotes the standard multivariate normal cumulative distribution:

$$
\Phi_{\Sigma}\left(x_{1}, \ldots, x_{n}\right)=\int_{-\infty}^{x_{1}} \ldots \int_{-\infty}^{x_{n}} \frac{1}{(2 \pi)^{n / 2}|\Sigma|^{1 / 2}} \exp \left(-\frac{1}{2} v^{\prime} \Sigma^{-1} v\right) d v
$$

where $v=\left(v_{1}, \ldots, v_{n}\right)$ and $\Sigma$ is a correlation matrix, that is symmetric, semi-definite positive with ones on the diagonal and off diagonal terms between -1 and 1 . The corresponding density is:

$$
c_{N}\left(u_{1}, \ldots, u_{n} ; \Sigma\right)=|\Sigma|^{-1 / 2} \exp \left[-\frac{1}{2}\left(x^{\prime} \Sigma^{-1} x-x^{\prime} x\right)\right],
$$

where $x=\left(\Phi^{-1}\left(u_{1}\right), \ldots, \Phi^{-1}\left(u_{n}\right)\right)$. The bivariate version that we use in the canonical vine copulas is:

$c_{\rho}\left(u_{1}, u_{2}\right)=\frac{1}{\sqrt{1-\rho^{2}}} \exp \left[\frac{-\left[\Phi^{-1}\left(u_{1}\right)^{2}+\Phi^{-1}\left(u_{2}\right)^{2}-2 \rho \Phi^{-1}\left(u_{1}\right) \Phi^{-1}\left(u_{2}\right)\right]}{2\left(1-\rho^{2}\right)}+\frac{\Phi^{-1}\left(u_{1}\right)^{2}+\Phi^{-1}\left(u_{2}\right)^{2}}{2}\right]$,

where $\rho$ is a correlation coefficient that lies between -1 and 1 .

The Gaussian copula has zero upper and lower tail dependence, $\lambda_{U}=\lambda_{L}=0$, except in the case of perfect correlation, $\rho=1$.

\subsubsection{Multivariate Student t copula}

The distribution function of the Student $t$ copula is:

$$
C_{T}\left(u_{1}, \ldots, u_{n} ; \Sigma, \nu\right)=T_{\Sigma, \nu}\left(T_{\nu}^{-1}\left(u_{1}\right), \ldots, T_{\nu}^{-1}\left(u_{n}\right)\right),
$$

where $T_{\nu}^{-1}(v)$ is the inverse of the cumulative distribution function of the univariate Student $\mathrm{t}$ with $\nu$ degrees of freedom and $T_{\Sigma, \nu}$ is given by:

$$
T_{\Sigma, \nu}\left(x_{1}, \ldots, x_{n} ;\right)=\int_{-\infty}^{x_{1}} \ldots \int_{-\infty}^{x_{n}} \frac{\Gamma\left(\frac{\nu+n}{2}\right)}{\Gamma\left(\frac{\nu}{2}\right) \sqrt{(\pi \nu)^{n}}|\Sigma|}\left(1+\frac{v^{\prime} \Sigma^{-1} v}{\nu}\right)^{\frac{-\nu+n}{2}} d v,
$$

where $v=\left(v_{1}, \ldots, v_{n}\right)$ and $\Sigma$ is a correlation matrix, that is symmetric, semi-definite positive with ones on the diagonal and off diagonal terms between -1 and 1 . The corresponding density is: 


$$
c_{T}\left(u_{1}, \ldots, u_{n} ; \Sigma, \nu\right)=\frac{\Gamma\left(\frac{\nu+n}{2}\right)}{\Gamma\left(\frac{\nu}{2}\right) \sqrt{(\pi \nu)^{n}}|\Sigma|} \frac{1}{\prod_{i=1}^{n} f_{\nu}\left(T_{\nu}^{-1}\left(u_{i}\right)\right)}\left(1+\frac{x^{\prime} \Sigma^{-1} x}{\nu}\right)^{\frac{-\nu+n}{2}} d v,
$$

where $x=\left(\Phi^{-1}\left(u_{1}\right), \ldots, \Phi^{-1}\left(u_{n}\right)\right)$ and $f_{\nu}($.$) is the density of the Student t distribution$ with $\nu$ degrees of freedom, $\rho \in(-1,1)$ and $\nu>2$. The bivariate version that we use in the canonical vine copulas is:

$$
c_{T}\left(u_{1}, u_{2} ; \rho, \nu\right)=\Gamma\left(\frac{\nu+2}{2}\right) \frac{1+\left(\frac{T_{\nu}^{-1}\left(u_{1}\right)^{2}+T_{\nu}^{-1}\left(u_{2}\right)^{2}-2 \rho T_{\nu}^{-1}\left(u_{1}\right) T_{\nu}^{-1}\left(u_{2}\right)}{\nu\left(1-\rho^{2}\right)}\right)^{-\left(\frac{\nu+2}{2}\right)}}{f_{\nu}\left(T_{\nu}^{-1}\left(u_{1}\right)\right) f_{\nu}\left(T_{\nu}^{-1}\left(u_{2}\right)\right) \nu \Pi \Gamma\left(\frac{\nu}{2}\right) \sqrt{1-\rho^{2}}} .
$$

The Student $\mathrm{t}$ copula has the same lower and upper tail dependence for every pair of variables: $\lambda_{U}=\lambda_{L}=2 t_{\nu+1}\left(-\sqrt{\nu+1} \sqrt{\frac{1-\rho}{1+\rho}}\right)$.

\subsubsection{Bivariate Gumbel and Rotated Gumbel copula}

The Gumbel copula has the following distribution:

$$
C_{G}\left(u_{1}, u_{2}, \theta\right)=\exp \left(-\left(\left(-\log u_{1}\right)^{\theta}+\left(-\log u_{2}\right)^{\theta}\right)^{1 / \theta}\right),
$$

and the following density:

$c_{G}\left(u_{1}, u_{2}, \theta\right)=\frac{C_{G}\left(u_{1}, u_{2}, \theta\right)\left(\log u_{1} \cdot \log u_{2}\right)^{\theta-1}}{u_{1} u_{2}\left(\left(-\log u_{1}\right)^{\theta}+\left(-\log u_{2}\right)^{\theta}\right)^{2-1 / \theta}}\left(\left(\left(-\log u_{1}\right)^{\theta}+\left(-\log u_{2}\right)^{\theta}\right)^{1 / \theta}+\theta-1\right)$,

where $\theta \in[1, \infty)$.

We use the rotated version of the Gumbel defined as: $C_{R G}\left(u_{1}, u_{2}, \theta\right)=u_{1}+u_{2}-1+$ $C_{R G}\left(1-u_{1}, 1-u_{2}, \theta\right)$ and $c_{R G}\left(u_{1}, u_{2}, \theta\right)=c_{G}\left(1-u_{1}, 1-u_{2}, \theta\right)$. For the rotated version of the Gumbel, $\lambda_{L}=2-2^{1 / \theta}, \lambda_{U}=0$.

\subsubsection{Bivariate Clayton copula}

The Clayton copula has the following distribution

$$
C_{C}\left(u_{1}, u_{2} ; \theta\right)=\left(u_{1}^{-\theta}+u_{2}^{-\theta}-1\right)^{-1 / \theta},
$$

and the following density:

$$
c_{C}\left(u_{1}, u_{2} ; \theta\right)=(1+\theta)\left(u_{1} u_{2}\right)^{-\theta-1}\left(u_{1}^{-\theta}+u_{2}^{-\theta}-1\right)^{-2-1 / \theta},
$$

where $\theta \in[-1, \infty) \backslash 0$.

The Clayton copula has lower but not upper tail dependence: $\lambda_{L}=2^{-1 / \theta}, \lambda_{U}=0$. 


\section{Tables}

\begin{tabular}{lcccccc}
\hline \hline & Mean & Standard Deviation & Skewness & Kurtosis & Min & Max \\
\hline Germany & 0.14 & 3.27 & -0.53 & 6.23 & -17.73 & 15.17 \\
France & 0.18 & 2.83 & -0.34 & 5.73 & -12.26 & 13.07 \\
UK & 0.13 & 2.29 & -0.52 & 5.61 & -11.05 & 10.02 \\
US & 0.17 & 2.38 & -0.13 & 6.25 & -12.13 & 12.61 \\
Japan & -0.01 & 3.07 & 0.26 & 3.66 & -10.99 & 11.18 \\
\hline Brazil & 0.15 & 5.30 & -0.47 & 4.80 & -22.02 & 22.25 \\
Mexico & 0.23 & 4.45 & -0.34 & 6.17 & -23.89 & 18.89 \\
Argentina & 0.13 & 5.26 & 0.13 & 5.62 & -21.81 & 25.03 \\
Chile & 0.03 & 3.16 & -0.19 & 4.31 & -13.10 & 10.23 \\
\hline
\end{tabular}

Table 1: Summary Statistics

Descriptive statistics of weekly index returns for the five countries of the G5 and the four countries of Latin America. All returns are expressed in US dollars from January 3, 1995 to May 30, 2006, which corresponds to a sample of 596 observations 


\begin{tabular}{lccccc}
\hline \hline & Germany & France & UK & US & Japan \\
\hline Germany & 1.00 & & & & \\
France & 0.86 & 1.00 & & & \\
UK & 0.74 & 0.77 & 1.00 & & \\
US & 0.67 & 0.65 & 0.64 & 1.00 & \\
Japan & 0.41 & 0.43 & 0.38 & 0.36 & 1.00 \\
\hline
\end{tabular}

\begin{tabular}{lcccc}
\hline \hline & Brazil & Mexico & Argentina & Chile \\
\hline Brazil & 1.00 & & & \\
Mexico & 0.64 & 1.00 & & \\
Argentina & 0.56 & 0.56 & 1.00 & \\
Chile & 0.58 & 0.54 & 0.45 & 1.00 \\
\hline
\end{tabular}

Table 2: Unconditional Correlation, G5 and Latin America

Unconditional Pearson correlation between the weekly index returns for the five countries of the G5 as well as the four countries of Latin America. 


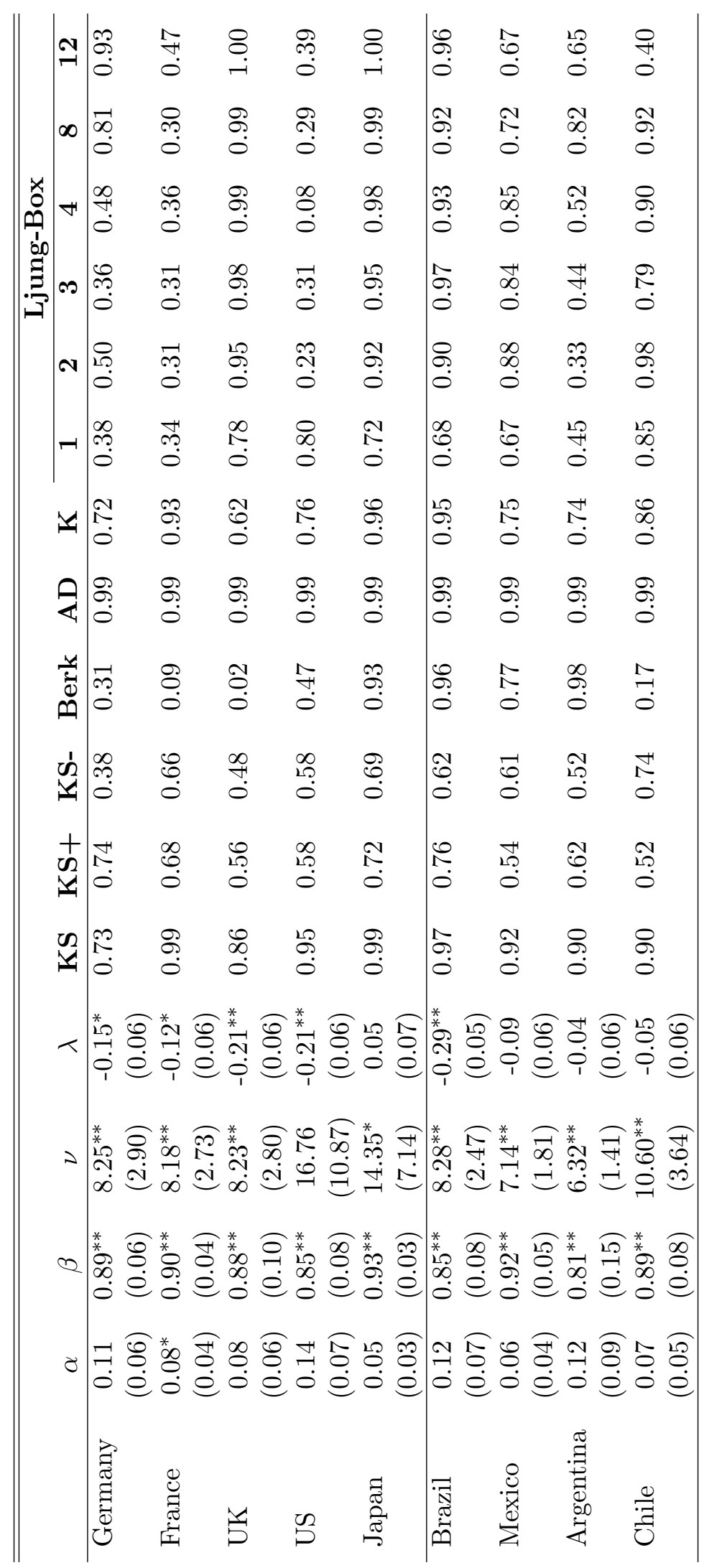

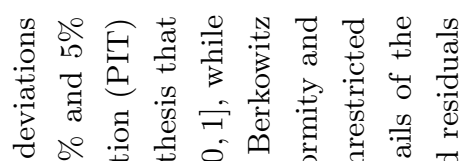

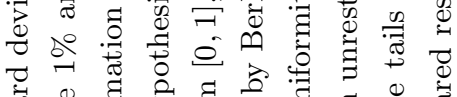

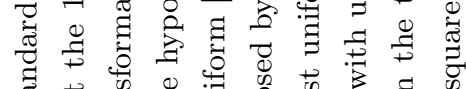

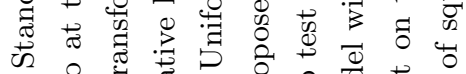
क

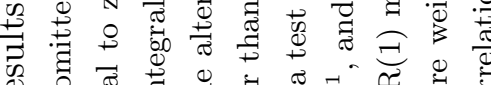

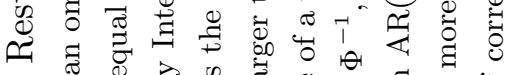

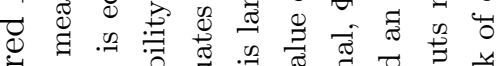

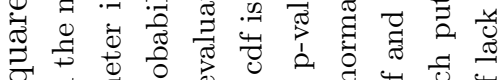
品

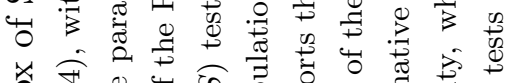

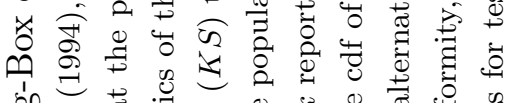

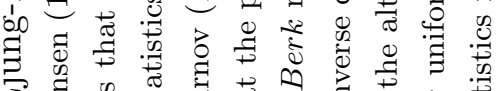

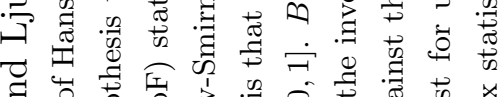

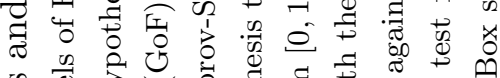

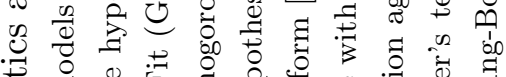

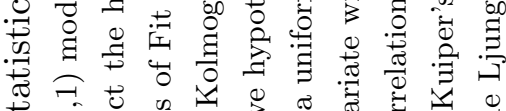

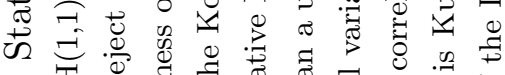

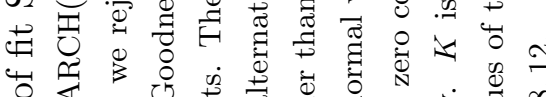

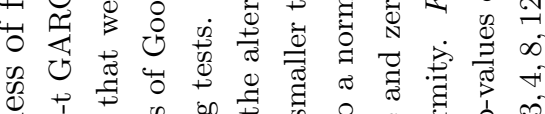

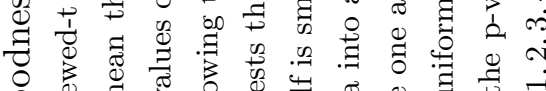

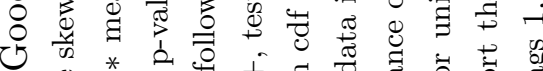

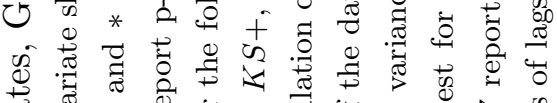

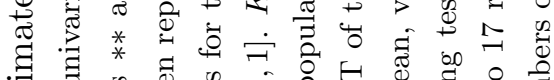

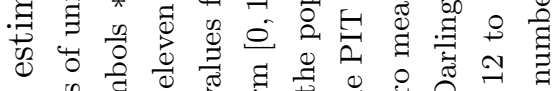

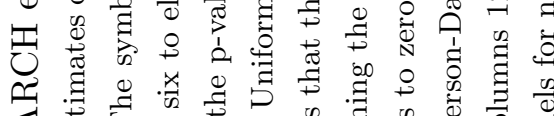

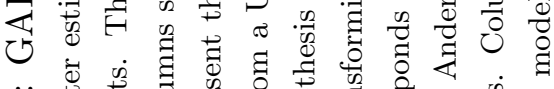

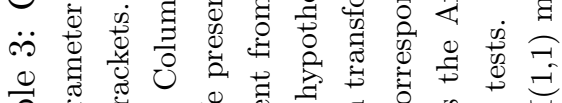

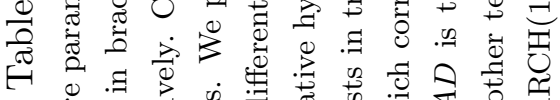

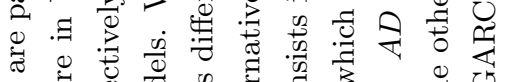

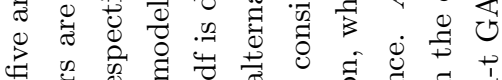

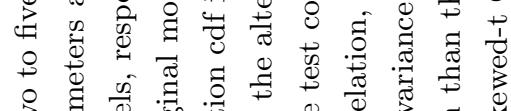

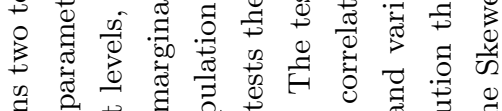

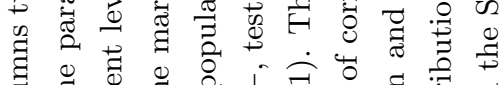

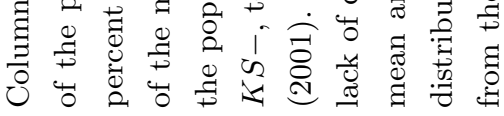




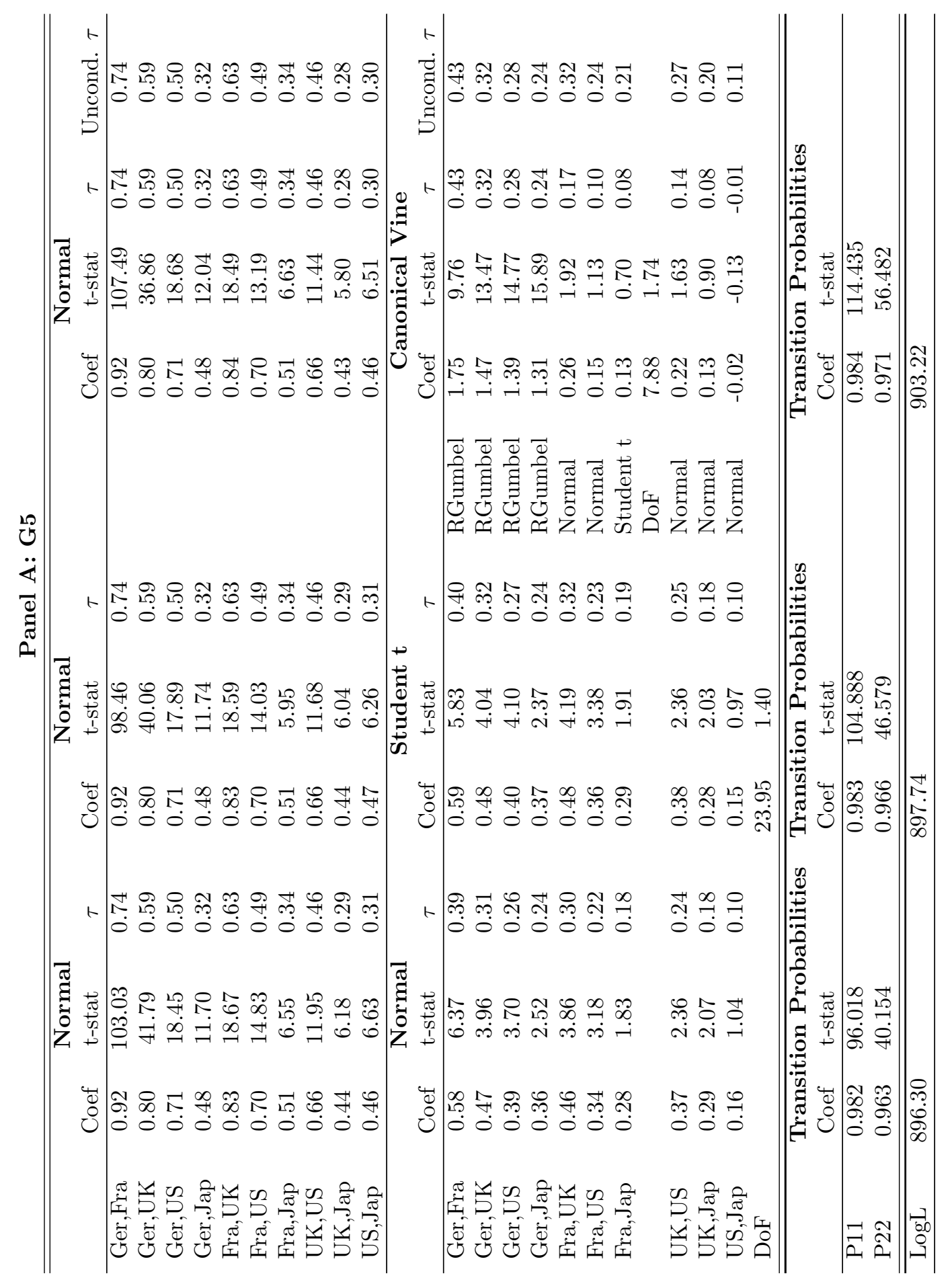




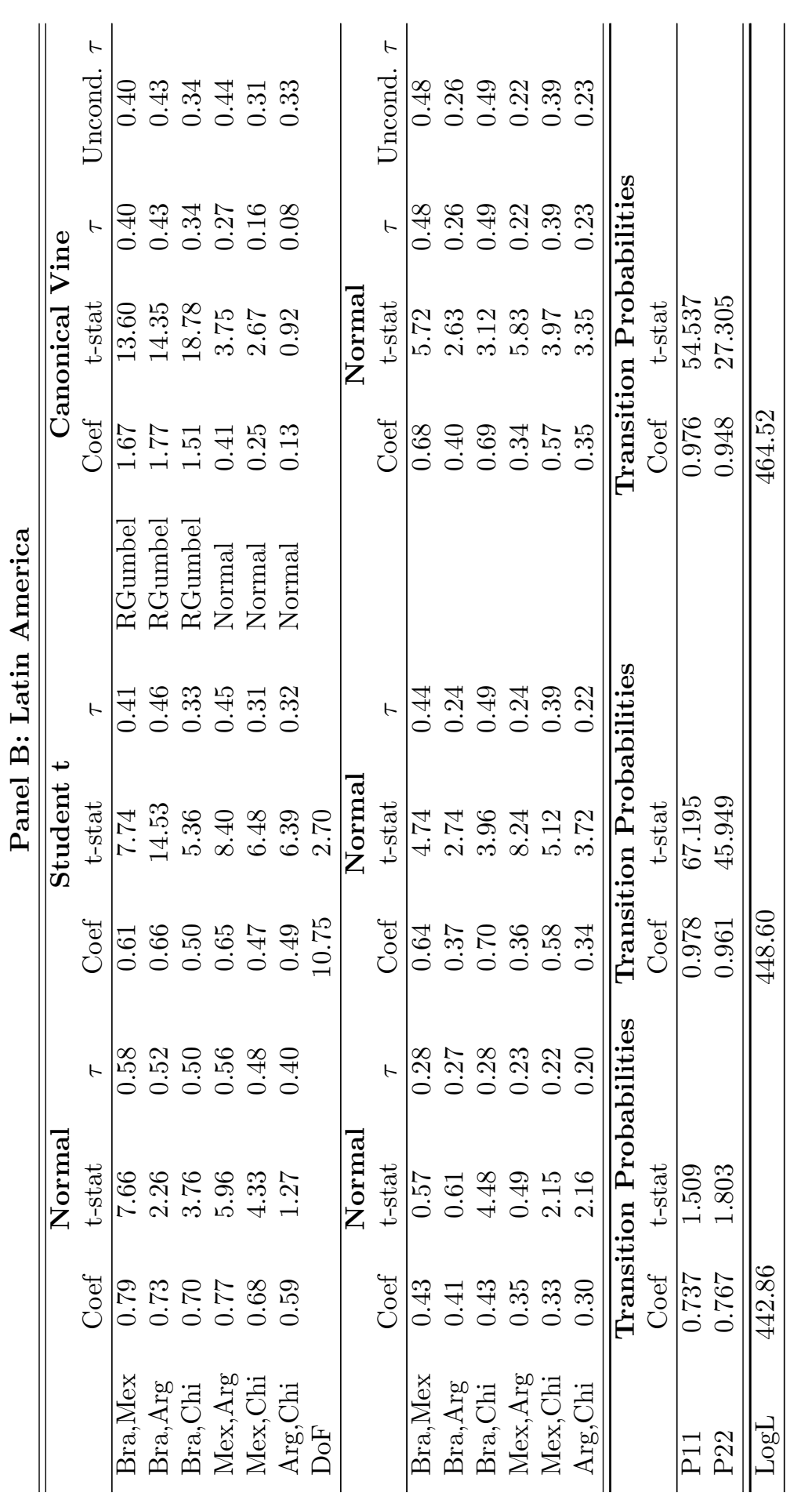

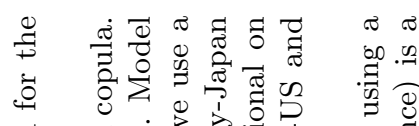

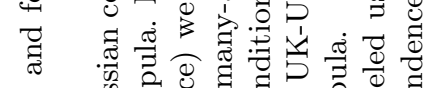

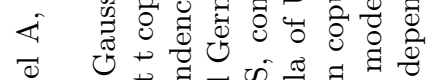
品 2.

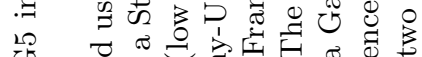
के

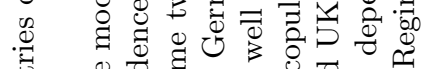

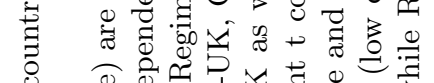

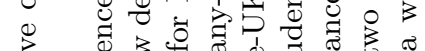

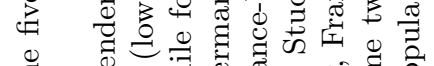

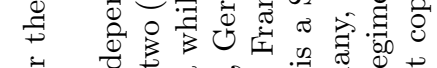
总 0 过宁 屯

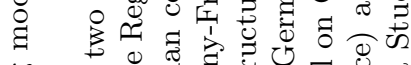

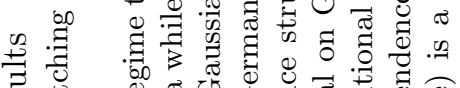

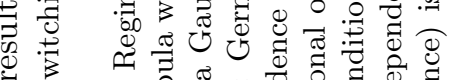
क \%

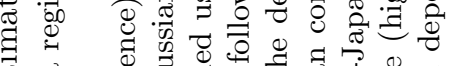

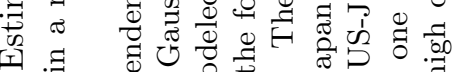
$\ddot{7}$ o

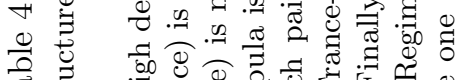

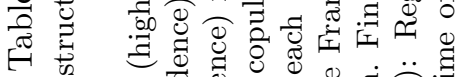

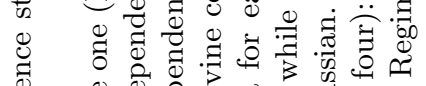

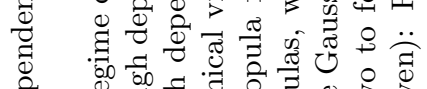

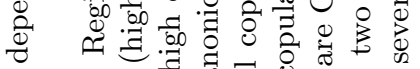

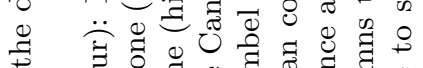

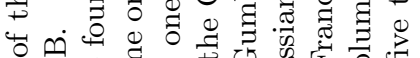
n

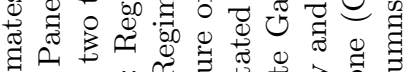

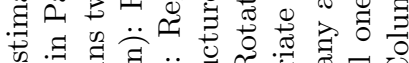
कै

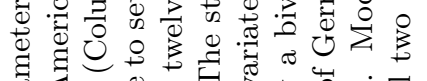

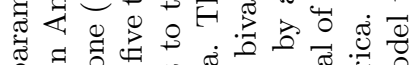
2. 可 总.

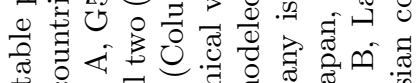

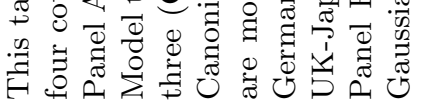




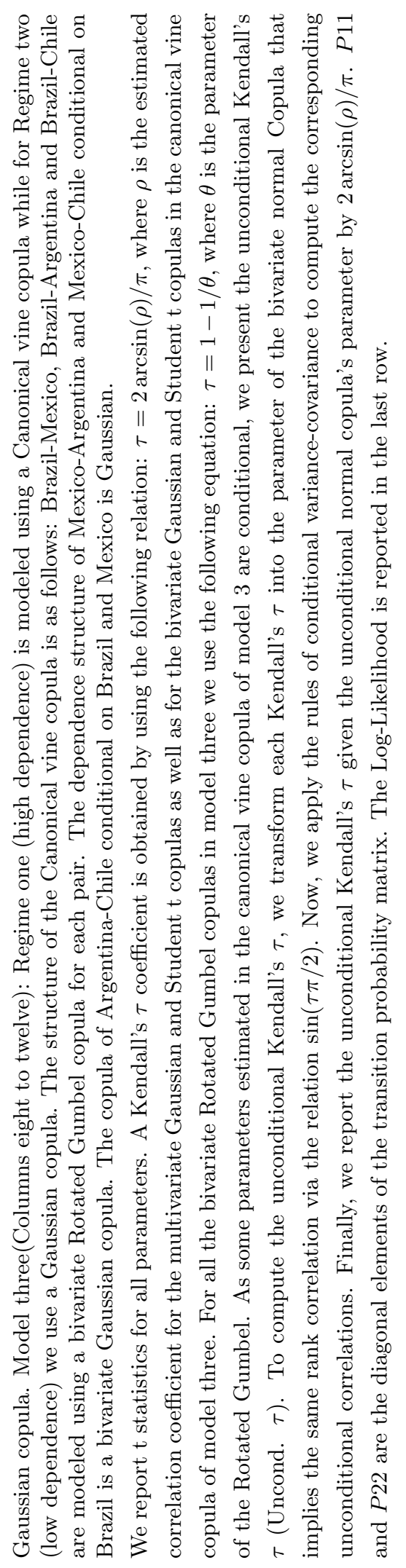




\section{$9 \quad$ Figures}

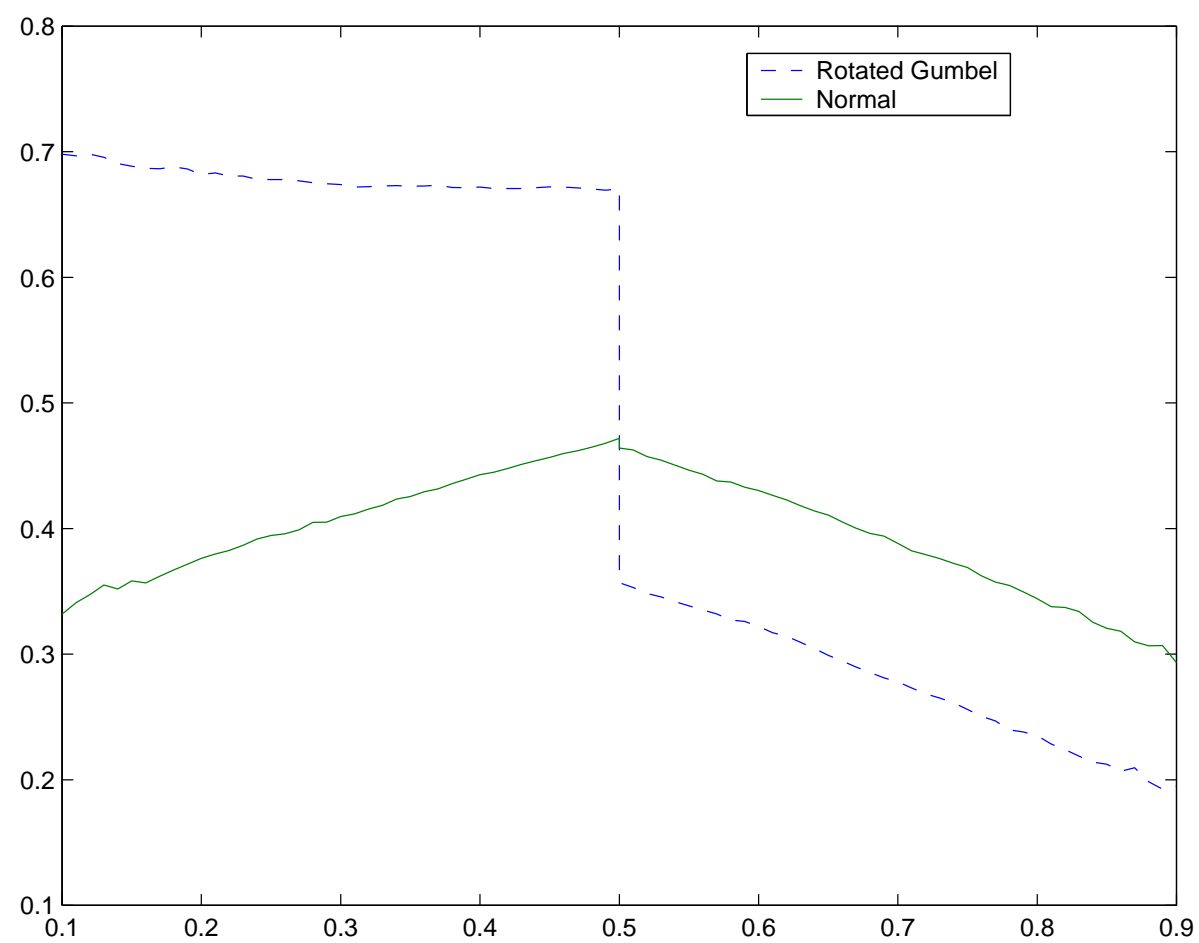

Figure 1: Exceedance Correlation from Gaussian and Rotated Gumbel copulas with standard normal marginals (100000 simulations, Kendall's $\tau=.5$ ). 

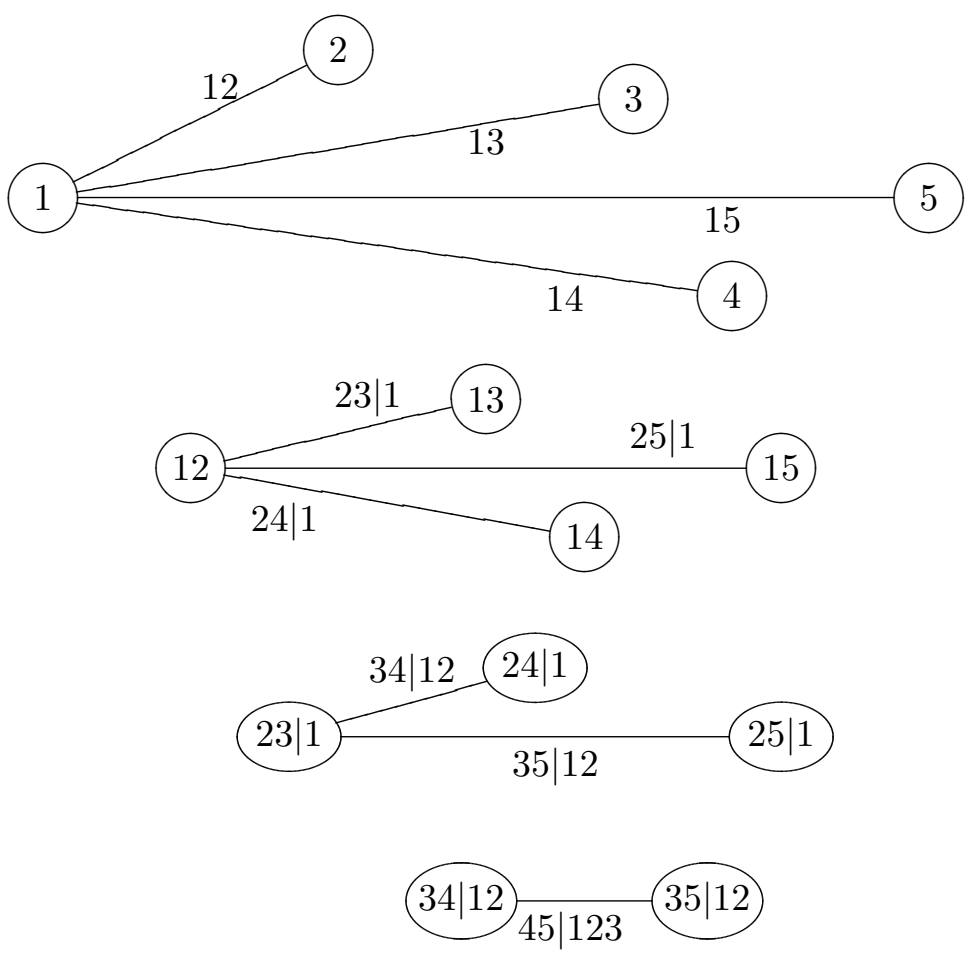

Figure 2: Dependence structure of a canonical vine

This figure shows the structure of a canonical vine copula with five variables. In the first layer, the dependence between variable 1 and all the other variables in the system is modeled with bivariate copulas. The second layer consists in modeling the dependence of variables 2 with variables 3 to 5 , conditionally on variable 1. In the last layer, one uses a bivariate copula to model the dependence between variables 4 and 5 , conditionally on variables 1 to 3 . In the case of this system with 5 variables, the dependence is modeled with 10 bivariate copulas 


$$
\begin{aligned}
& \theta_{i j \mid(1, \ldots, i-1)} \stackrel{(E q .4)}{\longrightarrow} \tau_{i j \mid(1, \ldots, i-1)} \stackrel{\sin (\tau \pi / 2)}{\longrightarrow} \rho_{i j \mid(1, \ldots, i-1)} \\
& \tau_{i j} \longleftarrow 2 \arcsin (\rho) / \pi \quad \rho_{i j}
\end{aligned}
$$

Figure 3: Computation of unconditional Kendall's $\tau$

This figure shows how we compute unconditional Kendall's $\tau, \tau_{i j}$ from $\theta_{i j \mid(1, \ldots, i-1)}$, the parameter of the conditional copula. First, we compute $\tau_{i j \mid(1, \ldots, i-1)}$, the Kendall's $\tau$ of each bivariate conditional copula implied by the estimated parameter using Equation (4). Second, we presume the data came form a Gaussian copula and we compute $\rho_{i j \mid(1, \ldots, i-1)}$, the correlation that implies the same Kendall's $\tau$, via the relation $\sin (\tau \pi / 2)$. Third, we apply the rules of conditional variance-covariance to compute $\rho_{i j}$, the corresponding unconditional correlations which we obtain by normalizing the unconditional variance covariance matrix obtained via the formula $\Sigma_{x \mid y}=\Sigma_{x}-\Sigma_{x y} \Sigma_{y}^{-1} \Sigma_{y x}$. Finally we report $\tau_{i j}$, the unconditional Kendall's $\tau$ that corresponds to the Gaussian unconditional correlation, with the relation $2 \arcsin (\rho) / \pi$. 

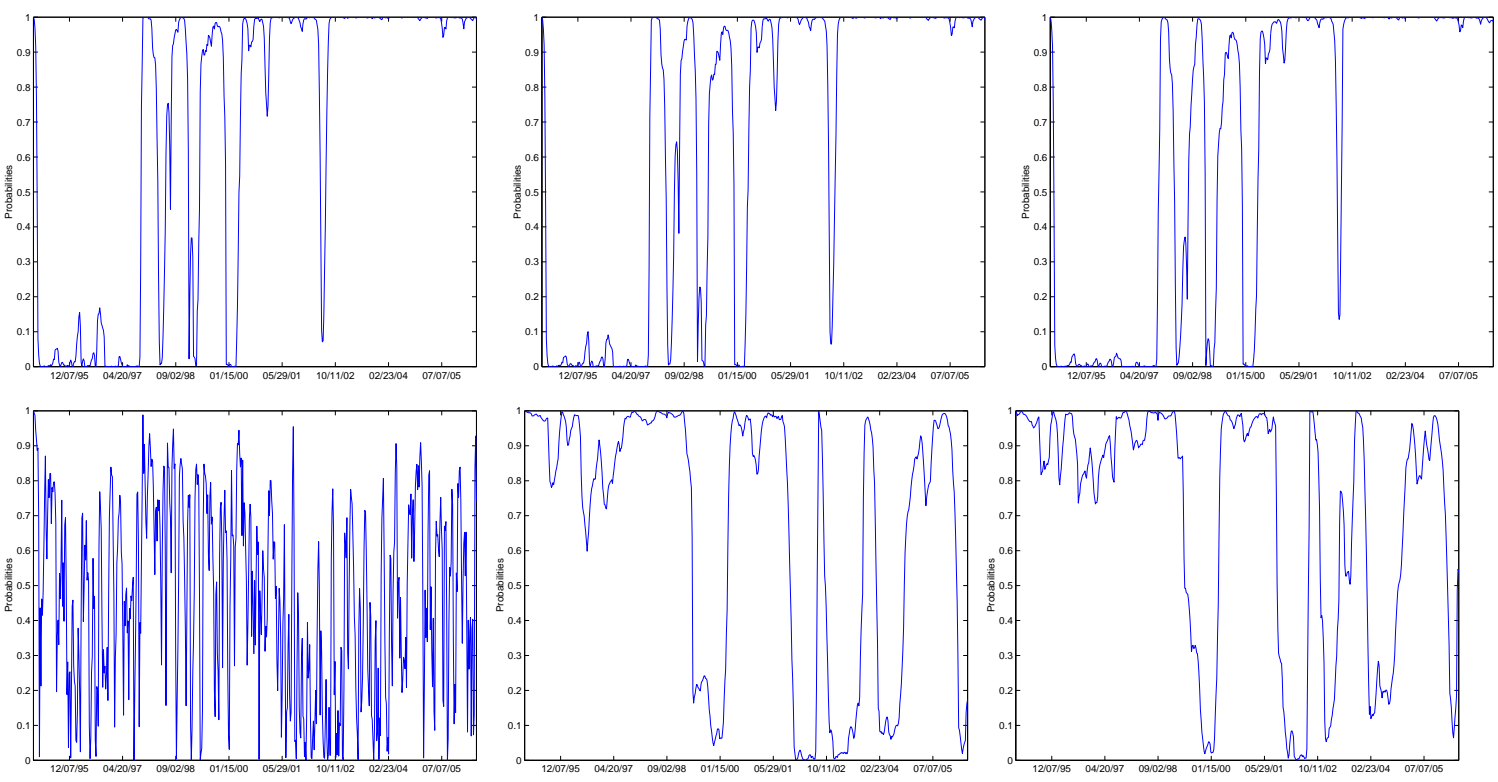

Figure 4: Smoothed probability of high dependence regime, G5 and Latin America. This figure presents the smoothed probability of the high dependence regime obtained from the EM algorithm for the G5 and Latin America. The first column corresponds to model 1, the Gaussian-Gaussian copula switching model. The second column is model 2, which corresponds to the Normal-Student t copula switching model for the G5, and the Student t-Normal for Latin America. The third column is the Normal-canonical vine copula switching model for G5, and the canonical vine-Normal copula for Latin America. 

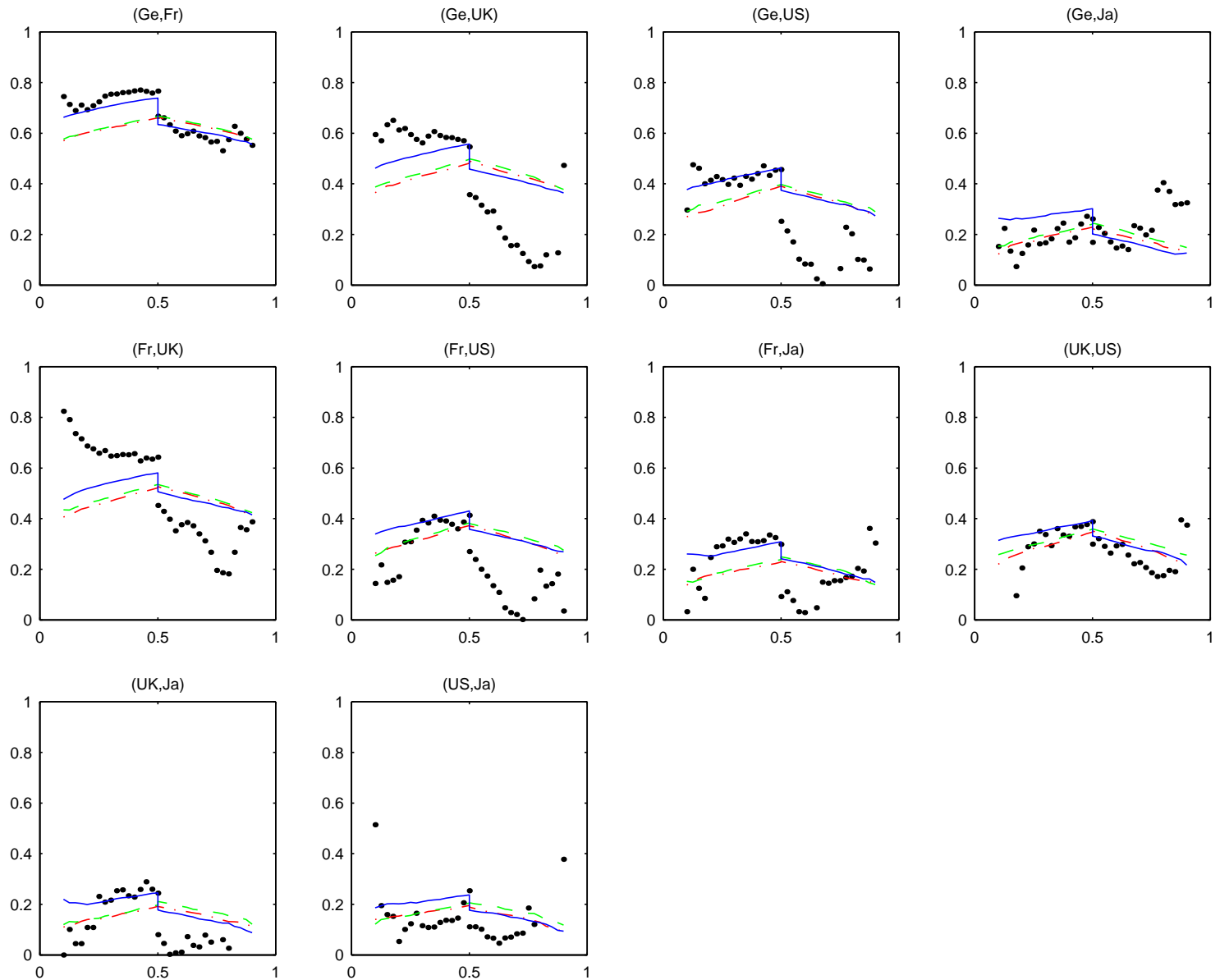

Figure 5: Exceedance correlation, data and models, G5 countries

This figure shows the pairwise empirical exceedance correlations of the G5 for the following thresholds: from 0.1 to 0.9 by increments of 0.025 . The exceedance correlation of the inverse normal cdf of the PIT of the marginals is represented by dots. The dashed line represents the all Gaussian copula model, the dot-dashed line represents the model with the Student-t copula, while the continuous line represents the canonical vine model. 

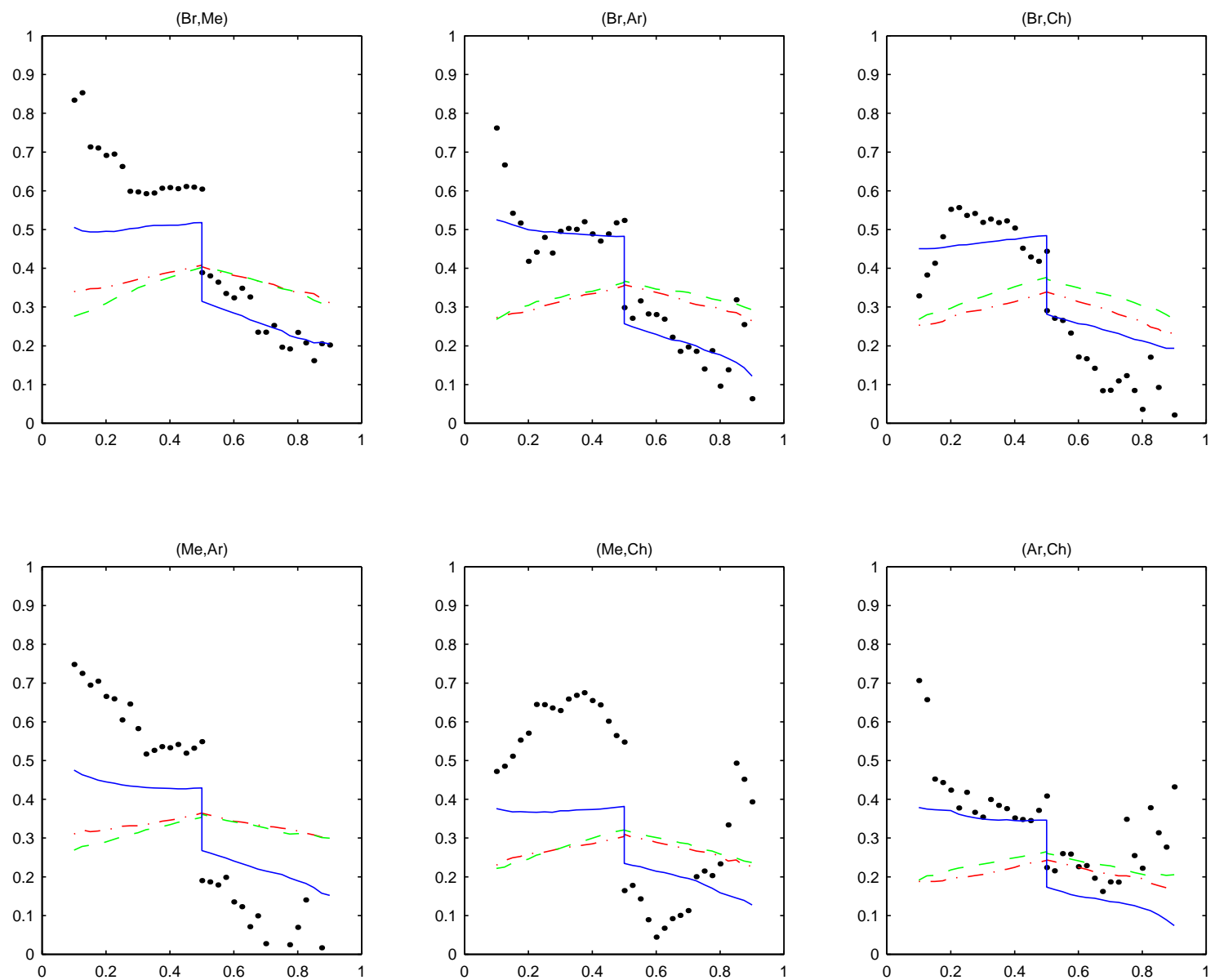

Figure 6: Exceedance correlation, data and models, Latin American countries

This figure shows the pairwise empirical exceedance correlations of Latin America for the following thresholds: from 0.1 to 0.9 by increments of 0.025 . The exceedance correlation of the inverse normal cdf of the PIT of the marginals is represented by dots. The dashed line represents the all Gaussian copula model, the dot-dashed line represents the model with the Student-t copula, while the continuous line represents the canonical vine model. 

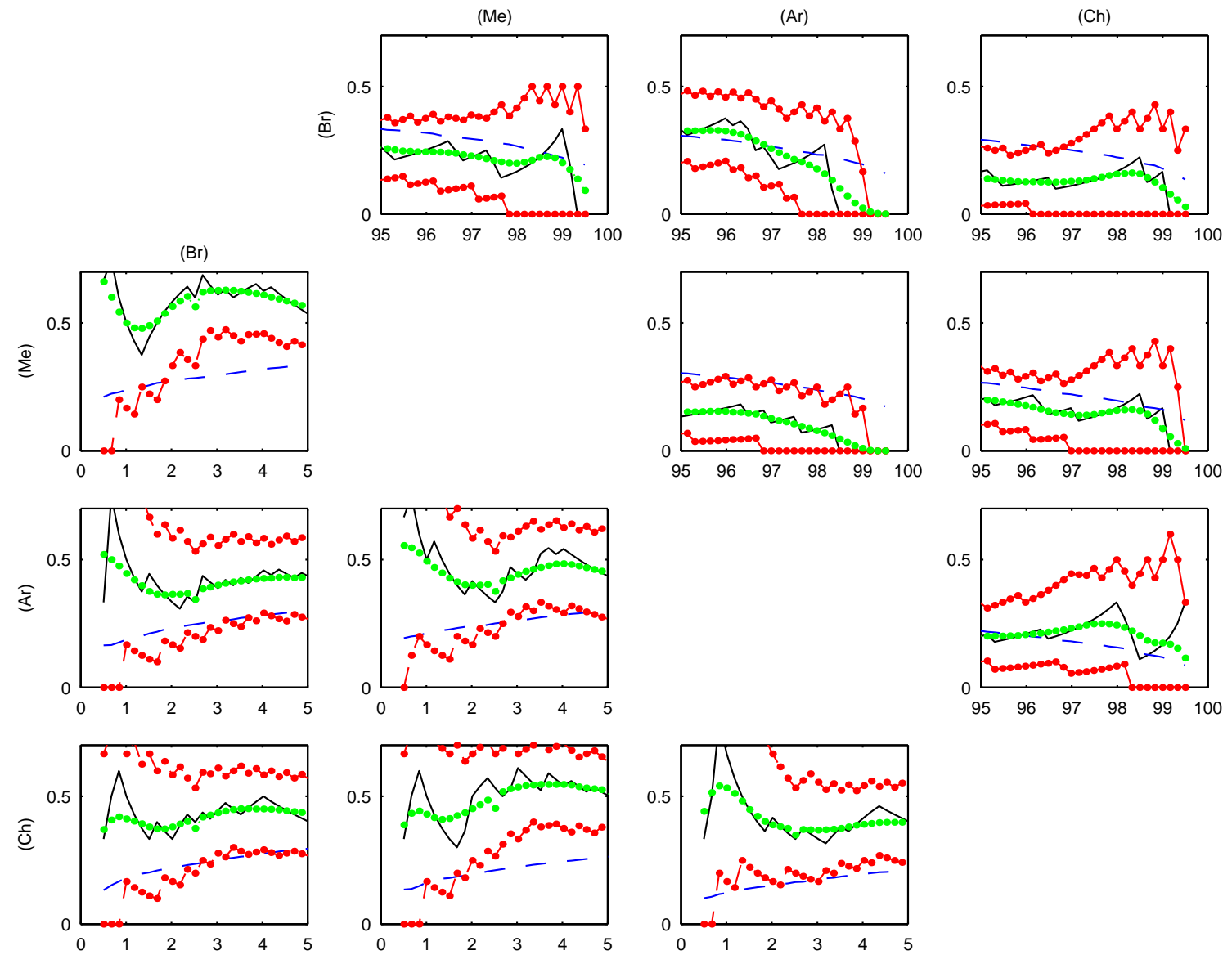

Figure 7: Quantile dependence, Gaussian-Gaussian model, with bootstrap confidence intervals based on the data, Latin American countries

This figure shows the pairwise quantile dependence implied by the PIT of the marginals and the all Gaussian copula model for Latin America. The continuous line represents the quantile dependence of the PIT of the marginals, while the dashed line is the one calculated from the simulations of the all Gaussian model. The $5 \%$ and $95 \%$ confidence intervals are drawn by lines connecting dots. These confidence intervals are obtained from 500 bootstrap replications of the data. The average over the bootstrap samples is represented by a dotted line. The graphs below the diagonal of each figure represent the lower quantile dependence, while the ones above the diagonal are the upper quantile dependence. 

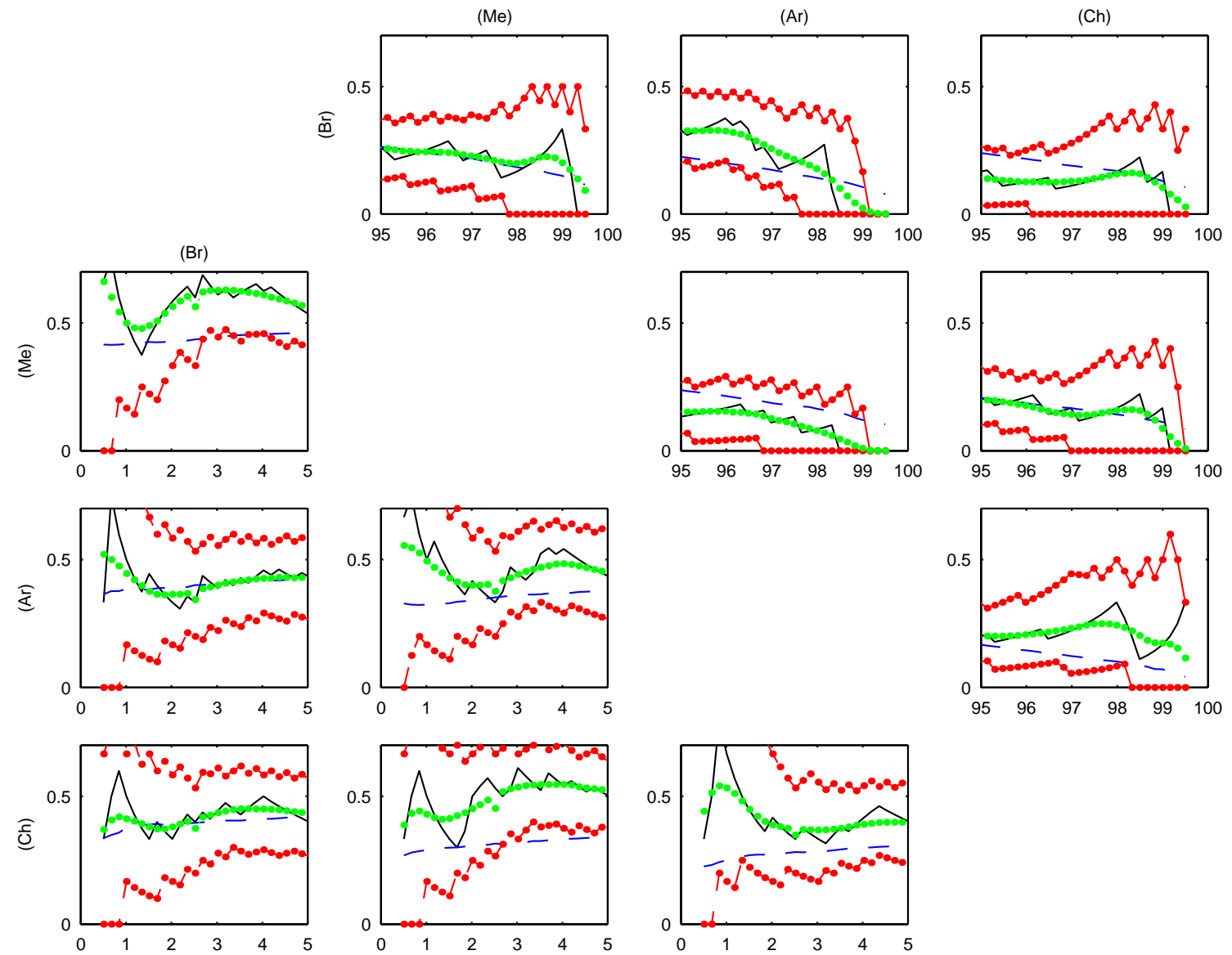

Figure 8: Quantile dependence, canonical vine-Gaussian model, with bootstrap confidence intervals based on the data, Latin American countries

This figure shows the pairwise quantile dependence implied by the PIT of the marginals and the canonical vine model for Latin America. The continuous line represents the quantile dependence of the PIT of the marginals, while the dashed line is the one calculated from the simulations of the canonical vine model. The $5 \%$ and $95 \%$ confidence intervals are drawn by lines connecting dots. These confidence intervals are obtained from 500 bootstrap replications of the data. The average over the bootstrap samples is represented by a dotted line. The graphs below the diagonal of each figure represent the lower quantile dependence, while the ones above the diagonal are the upper quantile dependence. 


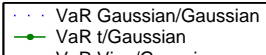

VaR t/Gaussian

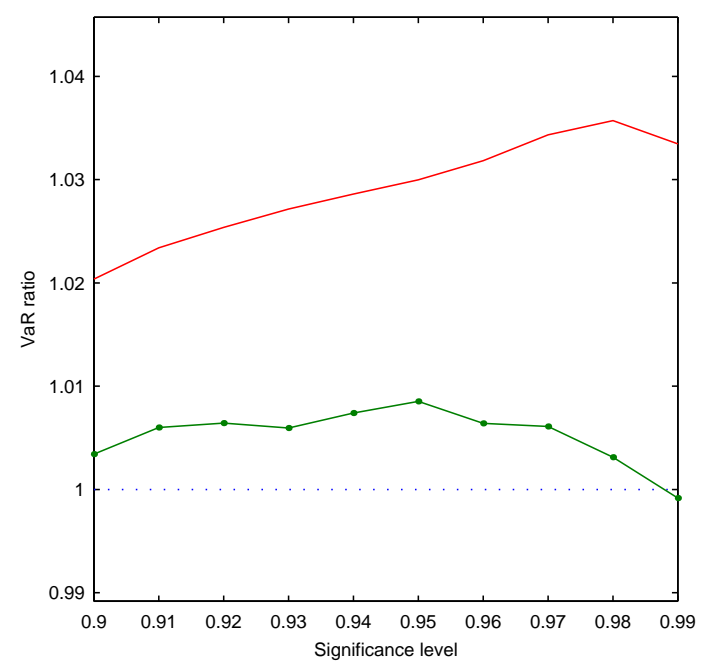

- ESR Gaussian/Gaussian

ESR t/Gaussian

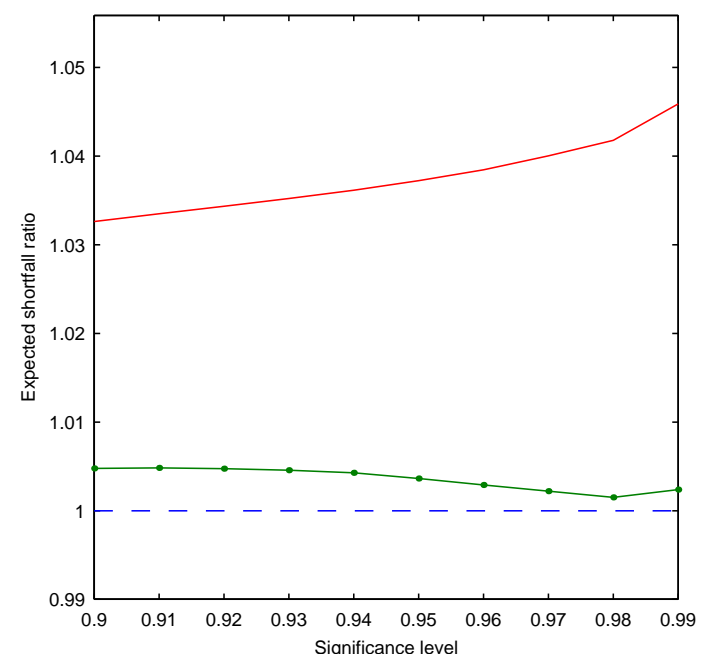

Figure 9: Expected Shortfall and Value at Risk with respect to the all Gaussian copula model, G5 countries

This figure shows the VaR and ES of an equally weighted portfolio, assuming normal marginals, for the Student-t and canonical vine model as a fraction of the all Gaussian copula model for the G5. The significance levels go from 0.9 to 0.99 by increments of 0.05 . In order to calculate the VaR and ES, for each model we simulate a long series of $N_{b}=298000$ observations. 


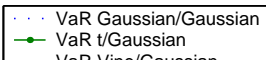

VaR t/Gaussian

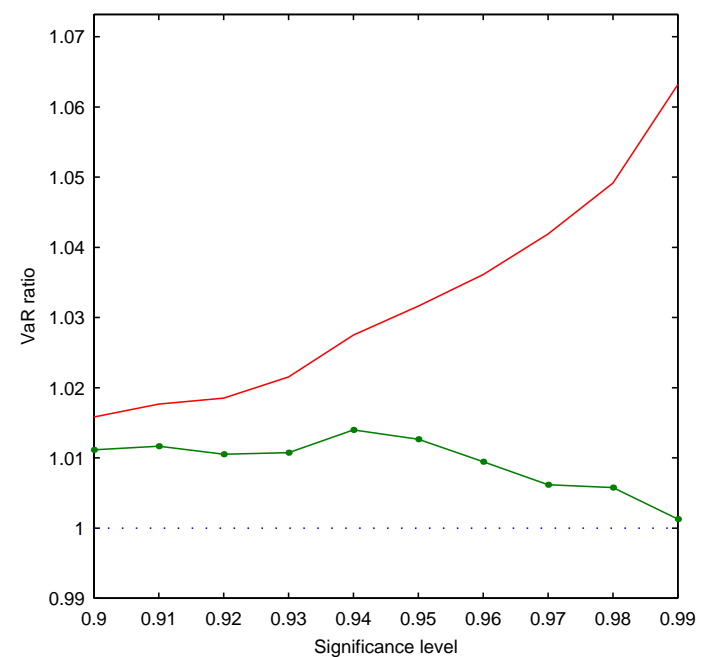

- ESR Gaussian/Gaussian

ESR t/Gaussian

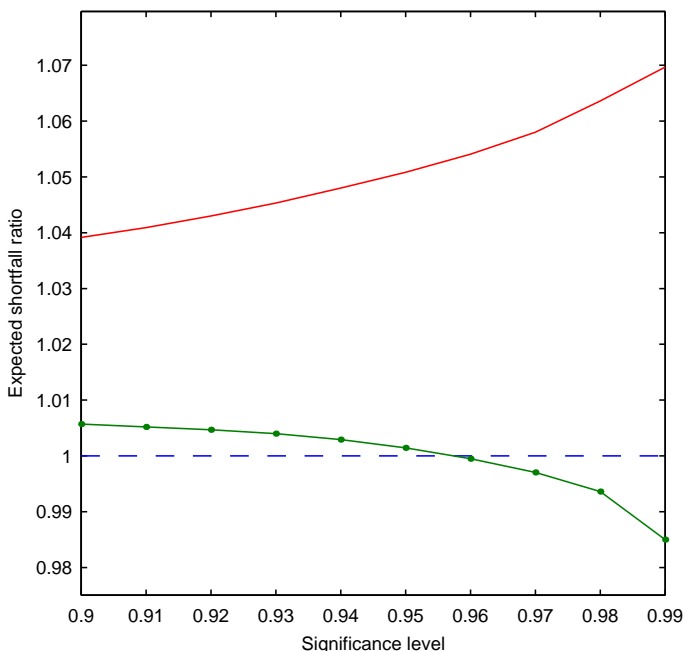

Figure 10: Expected Shortfall and Value at Risk with respect to the all Gaussian copula model, Latin American countries

This figure shows the VaR and ES of an equally weighted portfolio, assuming normal marginals, for the Student-t and canonical vine models as a fraction of the all Gaussian copula model for Latin America. The significance levels go from 0.9 to 0.99 by increments of 0.05 . In order to calculate the VaR and ES, for each model we simulate a long series of $N_{b}=298000$ observations. 


\section{Recent titles}

\section{CORE Discussion Papers}

2007/69. Pierre-André JOUVET, Pierre PESTIEAU and Gregory PONTHIERE. Longevity and environmental quality in an OLG model.

2007/70. Jean GABSZEWICZ, Didier LAUSSEL and Michel LE BRETON. The mixed strategy Nash equilibrium of the television news scheduling game.

2007/71. Robert CHARES and François GLINEUR. An interior-point method for the single-facility location problem with mixed norms using a conic formulation.

2007/72. David DE LA CROIX and Omar LICANDRO. 'The child is father of the man': Implications for the demographic transition.

2007/73. Jean J. GABSZEWICZ and Joana RESENDE. Thematic clubs and the supremacy of network externalities.

2007/74. Jean J. GABSZEWICZ and Skerdilajda ZANAJ. A note on successive oligopolies and vertical mergers.

2007/75. Jacques H. DREZE and P. Jean-Jacques HERINGS. Kinky perceived demand curves and Keynes-Negishi equilibria.

2007/76. Yu. NESTEROV. Gradient methods for minimizing composite objective function.

2007/77. Giacomo VALLETTA. A fair solution to the compensation problem.

2007/78. Claude D'ASPREMONT, Rodolphe DOS SANTOS FERREIRA and Jacques THEPOT. Hawks and doves in segmented markets: a formal approach to competitive aggressiveness.

2007/79. Claude D'ASPREMONT, Rodolphe DOS SANTOS FERREIRA and Louis-André GERARDVARET. Imperfect competition and the trade cycle: guidelines from the late thirties.

2007/80. Andrea SILVESTRINI. Testing fiscal sustainability in Poland: a Bayesian analysis of cointegration.

2007/81. Jean-François MAYSTADT. Does inequality make us rebel? A renewed theoretical model applied to South Mexico.

2007/82. Jacques H. DREZE, Oussama LACHIRI and Enrico MINELLI. Shareholder-efficient production plans in a multi-period economy.

2007/83. Jan JOHANNES, Sébastien VAN BELLEGEM and Anne VANHEMS. A unified approach to solve ill-posed inverse problems in econometrics.

2007/84. Pablo AMOROS and M. Socorro PUY. Dialogue or issue divergence in the political campaign?

2007/85. Jean-Pierre FLORENS, Jan JOHANNES and Sébastien VAN BELLEGEM. Identification and estimation by penalization in nonparametric instrumental regression.

2007/86. Louis EECKHOUDT, Johanna ETNER and Fred SCHROYEN. A benchmark value for relative prudence.

2007/87. Ayse AKBALIK and Yves POCHET. Valid inequalities for the single-item capacitated lot sizing problem with step-wise costs.

2007/88. David CRAINICH and Louis EECKHOUDT. On the intensity of downside risk aversion.

2007/89. Alberto MARTIN and Wouter VERGOTE. On the role of retaliation in trade agreements.

2007/90. Marc FLEURBAEY and Erik SCHOKKAERT. Unfair inequalities in health and health care.

2007/91. Frédéric BABONNEAU and Jean-Philippe VIAL. A partitioning algorithm for the network loading problem.

2007/92. Luc BAUWENS, Giordano MION and Jacques-François THISSE. The resistible decline of European science.

2007/93. Gaetano BLOISE and Filippo L. CALCIANO. A characterization of inefficiency in stochastic overlapping generations economies.

2007/94. Pierre DEHEZ. Shapley compensation scheme.

2007/95. Helmuth CREMER, Pierre PESTIEAU and Maria RACIONERO. Unequal wages for equal utilities.

2007/96. Helmuth CREMER, Jean-Marie LOZACHMEUR and Pierre PESTIEAU. Collective annuities and redistribution.

2007/97. Mohammed BOUADDI and Jeroen V.K. ROMBOUTS. Mixed exponential power asymmetric conditional heteroskedasticity. 


\section{Recent titles}

\section{CORE Discussion Papers - continued}

2008/1. Giorgia OGGIONI and Yves SMEERS. Evaluating the impact of average cost based contracts on the industrial sector in the European emission trading scheme.

2008/2. Oscar AMERIGHI and Giuseppe DE FEO. Privatization and policy competition for FDI.

2008/3. Wlodzimierz SZWARC. On cycling in the simplex method of the Transportation Problem.

2008/4. John-John D'ARGENSIO and Frédéric LAURIN. The real estate risk premium: A developed/emerging country panel data analysis.

2008/5. Giuseppe DE FEO. Efficiency gains and mergers.

2008/6. Gabriella MURATORE. Equilibria in markets with non-convexities and a solution to the missing money phenomenon in energy markets.

2008/7. Andreas EHRENMANN and Yves SMEERS. Energy only, capacity market and security of supply. A stochastic equilibrium analysis.

2008/8. Géraldine STRACK and Yves POCHET. An integrated model for warehouse and inventory planning.

2008/9. Yves SMEERS. Gas models and three difficult objectives.

2008/10. Pierre DEHEZ and Daniela TELLONE. Data games. Sharing public goods with exclusion.

2008/11. Pierre PESTIEAU and Uri POSSEN. Prodigality and myopia. Two rationales for social security.

2008/12. Tim COELLI, Mathieu LEFEBVRE and Pierre PESTIEAU. Social protection performance in the European Union: comparison and convergence.

2008/13. Loran CHOLLETE, Andréas HEINEN and Alfonso VALDESOGO. Modeling international financial returns with a multivariate regime switching copula.

\section{Books}

Y. POCHET and L. WOLSEY (eds.) (2006), Production planning by mixed integer programming. New York, Springer-Verlag.

P. PESTIEAU (ed.) (2006), The welfare state in the European Union: economic and social perspectives. Oxford, Oxford University Press.

H. TULKENS (ed.) (2006), Public goods, environmental externalities and fiscal competition. New York, Springer-Verlag.

V. GINSBURGH and D. THROSBY (eds.) (2006), Handbook of the economics of art and culture. Amsterdam, Elsevier.

J. GABSZEWICZ (ed.) (2006), La différenciation des produits. Paris, La découverte.

L. BAUWENS, W. POHLMEIER and D. VEREDAS (eds.) (2008), High frequency financial econometrics: recent developments. Heidelberg, Physica-Verlag.

P. VAN HENTENRYCKE and L. WOLSEY (eds.) (2007), Integration of AI and OR techniques in constraint programming for combinatorial optimization problems. Berlin, Springer.

\section{CORE Lecture Series}

C. GOURIÉROUX and A. MONFORT (1995), Simulation Based Econometric Methods.

A. RUBINSTEIN (1996), Lectures on Modeling Bounded Rationality.

J. RENEGAR (1999), A Mathematical View of Interior-Point Methods in Convex Optimization.

B.D. BERNHEIM and M.D. WHINSTON (1999), Anticompetitive Exclusion and Foreclosure Through Vertical Agreements.

D. BIENSTOCK (2001), Potential function methods for approximately solving linear programming problems: theory and practice.

R. AMIR (2002), Supermodularity and complementarity in economics.

R. WEISMANTEL (2006), Lectures on mixed nonlinear programming. 